Math. Model. Nat. Phenom.

Vol. 9, No. 4, 2014, pp. 38-64

DOI: $10.1051 / \mathrm{mmnp} / 20149404$

\title{
Optimal Sustainable Policies Under Pollution Ceiling: the Demographic Side
}

\author{
R. Boucekkine ${ }^{1}$, B. Martinez ${ }^{2}$, J.R. Ruiz-Tamarit ${ }^{3}$ \\ ${ }^{1}$ Aix-Marseille University (Aix-Marseille School of Economics), CNRS and EHESS, France \\ ${ }^{2}$ Department of Quantitative Economics, Universidad Complutense de Madrid, Spain \\ ${ }^{3}$ Department of Economic Analysis, Universitat de València, Spain, and IRES Department of \\ Economics, Université Catholique de Louvain, Belgium
}

\begin{abstract}
We study optimal sustainable policies in a benchmark logistic world (where both population and technological progress follow logistic laws of motion) subject to a pollution ceiling. The main policy in the hands of the benevolent planner is pollution abatement, ultimately leading to the control of a dirtiness index as in the early literature of the limits to growth literature. Besides inclusion of demographic dynamics, we also hypothesize that population size affects negatively the natural regeneration or assimilation rate, as a side product of human activities (like increasing pollution, deforestation,...). We first characterize optimal sustainable policies. Under certain conditions, the planner goes to the pollution ceiling value and stays on, involving a more stringent environmental policy and a sacrifice in terms of consumption per capita. Second, we study how the sustainable problem is altered when we depart from the logistic world by considering exponential technical progress (keeping population growth logistic). It's shown that, as expected, introducing such an asymmetry widens the margins of optimal policies as sustainable environmental policies are clearly less stringent under exponential technical progress. Third, we connect our model to the data, using in particular UN population projections.
\end{abstract}

Keywords and phrases: limits to growth, sustainable policy, optimal growth, demographic dynamics, pollution ceiling

Mathematics Subject Classification: 91B62, 91B76, 49J15

\section{Introduction}

Since the publication of the Meadows report in 1972, the long-term sustainability of the current human development standards has become a key debate in so many disciplines such as ecology and environmental science, climatology or economics. First, dismissed by leading economists like Nobel prize Solow (1974), the Meadows report and the limits to growth hypothesis have had an increasing importance over time in the economic literature. The issue of the long-term sustainability of the so-called modern economic growth regime is now at the heart of many economic research programs. Initially, the sustainability issue has been more tightly connected with the availability of natural resources, notably nonrenewable

\footnotetext{
${ }^{*}$ Corresponding author. E-mail: raouf.boucekkine@univ-amu.fr
} 
resources, rather than with the impact of carbon emissions on global warming and the like. An early sake for an operational sustainability concept can be found indeed in Hartwick (1977) who proposed an investment rule of rents extracted from the exploitation of nonrenewable resources to ensure a constant positive welfare level forever. Since then, many authors have attempted to build up normative and positive frames for sustainable development. Pioneering works in this stream are Barbier (1987), Daly (1990) and Pezzey (1992), while Chichilnisky (1996) has provided with an influential axiomatic approach to sustainability.

Environmental concerns, say pollution and global warming, came a bit later. As excellently explained in a recent article by Amigues and Moreaux (2013), the economists community responded to the first alarming reports of the IPCC (Intergovernmental Panel on Climate Change, launched in 1988) by constructing large scale models mimicking climatological models and therefore fully resorting to numerical simulations: these are the first integrated models merging economic and geophysical equations such like the DICE model developed by Nordhaus (1993). More economic approaches to sustainability under global warming have however emerged from the mid-90s. Many of the works in this stream combine the sustainability concern arising from depletion of (non-renewable) natural resources and the sustainability constraints due to environmental criteria (see, among others, Tahvonen and Kuuluvainen, 1993, and Withagen, 1994). Obviously, the two aspects are connected: carbon emissions come from the use of fossil energy and the nonrenewable natural resources studied in this literature are precisely fossil inputs. Imposing environmental norms like pollution ceilings constrains the dynamics of resource depletion, and therefore leads to decrease consumption at a certain point in time and a subsequent welfare loss. A large body of papers have been written along these lines as documented in Amigues and Moreaux (2013).

Strangely enough, population dynamics are typically ignored in this literature. In 1991, Robert Solow concluded a public lecture as follows: "...I have left out of this talk, as some of you may have noticed until now, any mention to population growth; and I did that on purpose, although it might be the natural first order concern if you are thinking about sustainability issues... You know that, I know that, and I have no particular competence to discuss it any further." (page 186). Indeed, there are several channels through which population growth impacts (sustainable) development: increasing depletion of natural resources, increasing pollution and other direct consequences of human activities (like deforestation, urbanization or intensive agriculture) which damage the self-cleaning capacity (or natural regeneration) of Nature. In this paper, we propose a benchmark demo-economic model which incorporates some realistic demographic ingredients and still allows for a (limited) analytical characterization. In particular, we assume two things. First of all, we hypothesize that population follows an exogenous logistic law of motion. Clearly enough, the logistic law is only an approximation (and probably a rough one in many circumstances) to human demographic dynamics. This said, it clearly outperforms the exponential growth law of motion typical assumed in the neoclassical economic growth theory which is by far unrealistic as it assumes unbounded growth. Recently, several authors have attempted to incorporate bounded demographic growth into textbook economic growth models, the most successful being Guerrini (2006). Our paper can be seen as a similar incursion into the body of sustainable (optimal) growth models with pollution ceiling. Second, and beside the fact that a larger population means more aggregate production and consumption (just like in the neoclassical model with exponential growth), we further assume that a larger population size decreases the regeneration rate of Nature. Again, this might be a direct consequence of urbanization and deforestation, or via an increase in pollution. Several authors have been arguing since Tahvonen and Withagen (1996) that the regeneration rate falls in an irreversible way if the pollution stock exceeds a certain threshold value (see also Prieur, 2009, and Boucekkine et al., 2013a, 2013b). We shall assess whether these two "demographic" departures from the typical sustainable growth model matter in the design of optimal policies and to which extent they do matter. It's worth pointing out here that we do not include in this benchmark analysis population control policies. In our framework, population dynamics are exogenous (following a logistic curve as mentioned just above). Rather, we complement our theoretical analysis with some numerical simulations based on UN demographic projections: with these 
projections in hands, should we expect that pollution ceilings will be soon or later reached, ultimately requiring the tightening of environmental regulation standards?

In order to derive some analytical results, we remove natural resources depletion from the model. Since population growth is already active through the natural regeneration rate, the model already includes a significant channel through which demographic growth affects the environment, and the size of this effect can be calibrated in the associated numerical experiments. With this simplification, the considered optimal growth model includes two state equations (capital and pollution) and two exogenously given law of motions for population and technology, the pollution stock being subject to a ceiling (pure state constraint). To keep some symmetry in the benchmark model, we shall start modelling technological dynamics as a logistic curve too (bounded growth) to neutralize somehow the exogenous pro and contrasustainable forces. The case of exogenous exponential technological progress is also studied to complement the analysis. Needless to say, just like for population dynamics, controlled technological dynamics are also another way to ensure sustainable growth via R\&D expenditures, either to foster output-increasing or energy-saving technological progress (see Boucekkine, Hritonenko and Yatsenko, 2013, for a recent framework including a pollution ceiling, or for the development of backstop technologies as in Tsur and Zemel, 2003). Again, here we focus on environmental policy. Environmental policy consists in a single control, abatement. To model abatement, we follow the line opened by Stokey (1998) as recently reformulated by Aznar and Ruiz-Tamarit (2012). Last but not least, as repeatedly mentioned above, we work within the framework of optimal growth, that's by considering a benevolent planner maximizing the social welfare of the economy subject to the set of "endogenous" and exogenous state equations listed above and in particular to a pollution ceiling. Welfare per period depends on consumption per capita and environmental quality pretty much in line with the traditional approach in this stream of literature (see Stokey, 1998, after Forster, 1975).

The article is organized as follows. Section 2 describes the benchmark economy. Section 3 presents the complete optimal growth model to study and derives the set of necessary conditions. Section 4 studies the non-binding state constraint case. Section 5 analyses the situation where the pollution ceiling is reached. Section 6 goes to the special case where technological progress has unbounded growth (exponential technological progress). In Sections 3 to 6, the long-term equilibria are derived in closed-form and discussed. Section 7 is the experimental part of the paper: the benchmark model is first calibrated, then simulated along three demographic scenarios based on UN projections. Section 8 concludes.

\section{The model economy}

The model economy is a one sector closed economy. Output is obtained according to an aggregate neoclassical production function depending on the technological level and both physical capital and labor stocks,

$$
Y(t)=A(t)^{1-\beta} K(t)^{\beta} N(t)^{1-\beta} .
$$

For the sake of simplicity, we assume that this production function comes from the direct summation of the individual production functions for many identical firms. The technical progress is Harrod-neutral. Technological level, denoted by $A$, is exogenous. In the benchmark analysis, we assume that it evolves according to the logistic differential equation

$$
\dot{A}(t)=\bar{x} A(t)\left(1-\frac{A(t)}{\bar{A}}\right),
$$

where $\bar{A}>0$ is the technological carrying capacity and $\bar{x} \geq 0$ is an upper bound for the rate of technological change. The initial technological level is $A\left(t_{0}\right)=A_{0}>0$. As mentioned in the introduction, this benchmark technological configuration is considered to keep a certain symmetry with population dynamics. The case of exponential technological growth, which is a limit case of the logistic curve, is handled 
in Section 6. It's worth mentioning here that while the logistic curves (also called $S$-shaped curves in the technological literature) better fit the diffusion of a given technology (reflecting for example learning or imitation), they may not be good at representing technological progress from a long-term perspective. Recently, Chang and Baek (2010) have studied technological improvements from this perspective: they show that the latter take the form of an upward trended sequence of connected or disconnected $S$-shaped curves, featuring a kind of sustainable improvement without limit mainly due to a series of emerging new technologies. Our study includes both the logistic and exponential cases.

The solution to the above Bernoulli's differential equation is

$$
A(t)=\frac{\bar{A} A_{0} \exp \left(\bar{x}\left(t-t_{0}\right)\right)}{A_{0} \exp \left(\bar{x}\left(t-t_{0}\right)\right)+\left(\bar{A}-A_{0}\right)} .
$$

We can see that $A$ monotonically increases converging to $\lim _{t \rightarrow \infty} A(t)=\bar{A}$. Consequently, the variable rate of technical progress is

$$
x(t)=\bar{x}\left(1-\frac{A(t)}{\bar{A}}\right)=\frac{\bar{x}\left(1-\frac{A_{0}}{\bar{A}}\right)}{\frac{A_{0}}{\bar{A}} \exp \left(\bar{x}\left(t-t_{0}\right)\right)+\left(1-\frac{A_{0}}{\bar{A}}\right)},
$$

with $x\left(t_{0}\right)=x_{0}=\bar{x}\left(1-\frac{A_{0}}{\bar{A}}\right) \leq \bar{x}$ and $\lim _{t \rightarrow \infty} x(t)=0$.

The economy is populated by many identical and infinitely lived agents. Population, denoted by $N$, is assumed exogenous but it evolves according to the logistic differential equation

$$
\stackrel{\bullet}{N}(t)=\bar{n} N(t)\left(1-\frac{N(t)}{\bar{N}}\right)
$$

where $\bar{N}>0$ is the population carrying capacity and $\bar{n} \geq 0$ is an upper bound for the rate of population growth. The initial population is $N\left(t_{0}\right)=N_{0}>0$. The solution to the above Bernoulli's differential equation is

$$
N(t)=\frac{\bar{N} N_{0} \exp \left(\bar{n}\left(t-t_{0}\right)\right)}{N_{0} \exp \left(\bar{n}\left(t-t_{0}\right)\right)+\left(\bar{N}-N_{0}\right)} .
$$

We can see that $N$ monotonically increases converging to $\lim _{t \rightarrow \infty} N(t)=\bar{N}$. Consequently, the variable rate of population growth is

$$
n(t)=\bar{n}\left(1-\frac{N(t)}{\bar{N}}\right)=\frac{\bar{n}\left(1-\frac{N_{0}}{\bar{N}}\right)}{\frac{N_{0}}{\bar{N}} \exp \left(\bar{n}\left(t-t_{0}\right)\right)+\left(1-\frac{N_{0}}{\bar{N}}\right)} .
$$

with $n\left(t_{0}\right)=n_{0}=\bar{n}\left(1-\frac{N_{0}}{\bar{N}}\right) \leq \bar{n}$ and $\lim _{t \rightarrow \infty} n(t)=0$.

We now come to a crucial part of the model, pollution and abatement modelling. Although pollution emissions may be originated both in the processes of consumption and production, we focus on pollution generated by firms. Pollution emissions are a by-product of economic activity, arising as an unintended output when firms produce according to (2.1). There is not free disposability of the residuals. Instead, firms are allowed to engage in costly abatement activities to mitigate emissions they produce, and the environment shows a certain absorptive capacity to clean up pollution. ${ }^{1}$

\footnotetext{
${ }^{1}$ We only consider mitigation in this paper. Alternative environmental policies include adaptation measures to global warming such like improvement of coastal protection infrastructure or warning systems (see Bréchet et al., 2013).
} 
Following Aznar and Ruiz-Tamarit (2012), we argue that all these issues are better captured in a multiple relationship framework. One major feature of our model is the existence of a stock of pollutants $S$, which is increased by polluting activities such as production $Y$, but is reduced by abatement $\mathcal{B}$ as well as by the corresponding natural regeneration at a variable rate $\delta(t) \geq 0$. Moreover, we require that pollution cannot exceed an upper bound for $S$, called $S^{\max }$, which thus plays the role of a ceiling value beyond which it is implicitly assumed that the economy enters a catastrophic state. The critical level $S^{\text {max }}>0$ is known with certainty, but the occurrence of the catastrophic event is endogenous and depends on agents' economic decisions. Under these assumptions, sustainable development will be characterized as a situation where the main economic variables show nonnegative long-run balanced growth while, at the same time, they contribute to generate an accumulated stock of pollutants smaller than (or equal to) the critical value $S^{\max }$.

The above-mentioned abatement effort $\mathcal{B}$, which is costly and decided by economic agents, will be measured in terms of output $Y(t)$ in such a way that these two variables relate to each other according to

$$
\mathcal{B}(t)=Y(t)-Y_{N}(t)=(1-z(t)) Y(t) .
$$

Here $z(t)$ represents, as in Stokey (1998), a measure of the effective dirtiness of the technique used to produce. Obviously, $z(t)=1-\frac{\mathcal{B}(t)}{Y(t)} \in[0,1]$ because resources devoted to clean pollution can never exceed the current production. Therefore, any choice for $z$ close to zero or one automatically makes the existing technique less or more polluting respectively. The above expression introduces a definition for the net output as

$$
Y_{N}(t)=z(t) Y(t) .
$$

The equation governing the motion of $S$ may be written as $\dot{S}(t)=P(Y(t), \mathcal{B}(t))-\delta(t) S(t)$, where $P(Y(t), \mathcal{B}(t))$ represents the net flow of waste and emissions associated with the endogenously determined levels of production-polluting and abatement activities. This flow is increasing with respect to $Y$ and decreasing with respect to $\mathcal{B}$, i.e., $P_{1}>0$ and $P_{2}<0$. Function $P($.$) is assumed homogeneous of$ degree zero, i.e., a proportionally equal increase in both output and abatement leaves net emissions unchanged independently of the population size. Consequently, the emissions function may be rewritten as $P(Y(t), \mathcal{B}(t))=G\left(\frac{\mathcal{B}(t)}{Y(t)}\right)$, where we assume strict concavity: $G^{\prime}<0, \lim _{x \rightarrow 0^{+}} G^{\prime} \leq 0,-\infty<\lim _{x \rightarrow 1^{-}} G^{\prime}<0$, $G^{\prime \prime}<0, G(0)=G^{M}>0$, and $G(1)=0$. Actually, $G^{M}>\bar{\delta} S^{\max }$ represents an effective upper limit for the emissions function, which is high enough to lead the economy, if it prevails, to the state of ecological catastrophe. Now, substituting the previous variable transformations into the differential equation for the motion of the stock of pollutants we get

$$
\dot{S}(t)=G(1-z(t))-\delta(t) S(t),
$$

where $G_{z}=-G^{\prime}>0,0<\lim _{z \rightarrow 0^{+}} G_{z}<+\infty, \lim _{z \rightarrow 1^{-}} G_{z} \geq 0$, and $G_{z z}=G^{\prime \prime}<0$. Taking as reference $z=1$, which implies that no abatement effort is done and that emissions flow reaches the maximum level $G^{M}$, the larger the reduction in $z$ the more effective the reduction in emissions. The initial pollution stock is $0<S\left(t_{0}\right)=S_{0}<S^{\max }$.

A particular strictly concave emissions function which may be used in numerical simulations is:

$$
G(1-z(t))=G^{M}-G^{M}(1-z(t))^{2}=G^{M} z(t)(2-z(t)) .
$$

Moreover, the natural rate of regeneration is not an exogenously given constant parameter but a variable which depends on the current population level. As explained in the introduction, this is a key channel through which demographic conditions affect the environment. Concretely, we hypothesize that the natural regeneration rate is strictly decreasing in the population level

$$
\delta(t)=\delta(N(t))
$$


being $\delta^{\prime}(N)<0$ together with $\delta\left(N_{0}\right)=\delta_{0}>\delta(\bar{N})=\bar{\delta} \geq 0$. In our framework, the regeneration rate reaches a limit lowest value $\bar{\delta}$ when population is maximal. This limit value need not be zero as population size is always bounded. Moreover, the limit value need not depend on $\bar{N}$ and may depend on many other several (unmodelled) long-term technological and ecological factors. We shall come back to this issue in next sections.

A particular affine function for the rate of regeneration which may be used in numerical simulations is

$$
\delta(N(t))=\left(\delta_{0}-\bar{\delta}\right)\left(\frac{\bar{N}-N(t)}{\bar{N}-N_{0}}\right)+\bar{\delta}=\frac{\left(\delta_{0}-\bar{\delta}\right) \bar{N}}{N_{0} \exp \left(\bar{n}\left(t-t_{0}\right)\right)+\left(\bar{N}-N_{0}\right)}+\bar{\delta} .
$$

According to the aggregate resources constraint, net output may be devoted to consumption or capital accumulation. For the sake of simplicity we do not consider capital depreciation. Hence, net investment equals gross investment and the capital stock is governed by the differential equation

$$
C(t)+\stackrel{\circ}{K}(t)=Y(t)-\mathcal{B}(t) .
$$

This equation also reflects the cost of the abatement activity in a very simple way: one additional unit of abatement effort is automatically 'transformed' into a lower unit of output available for consumption or capital accumulation. This particular 'one-to-one' transformation contributes to simplify our analysis.

Individual preferences are assumed to be represented by a twice continuously differentiable instantaneous utility function $V(c(t), P(t))$, which depends positively on the current per capita consumption $c$ and negatively on the polluting emissions flow $P$. Under this assumption, households do not care about the stock of pollutants $S$ accumulated in the environment and the atmosphere, but only about the current net flow of polluting waste and emissions $P$. This is because the local stock effect of pollution is assumed short-lived and the abatement activity, which reduces emissions and facilitates regeneration, makes it negligible. ${ }^{2}$ On the other hand, the assumed role for $S$ and $P$ in our model reflects the combined hypothesis of either discontinuous and continuous dependence of damages on pollution (the abrupt damage of a catastrophe via the stock $S$, and the marginal damage of pollution via the flow $P$ ); Hence, a non-zero marginal disutility shows that adverse effects occur even at pollution levels below the critical threshold.

As we have previously shown, emissions depend positively on production and negatively on pollution abatement, two variables that appear related to each other according to (2.5). Given the characterization of the emissions function, $P$ is an increasing monotonous transformation of $z$. Therefore, the instantaneous utility function may be written as $U(c(t), z(t))$ with $U_{c}>0$ and $U_{z}<0$, where the two ordinal utility functions $V$ and $U$ represent the same preference ordering. Moreover, we assume decreasing marginal utilities: $U_{c c}<0$ and $U_{z z}<0$, as well as strict concavity with respect to both arguments taken together, $U_{c c} U_{z z}-\left(U_{c z}\right)^{2}>0$.

The structure of the model allows for the existence of a long-run balanced growth path, defined as an allocation in which consumption per capita grows at a constant rate and the dirtiness index is constant.

\footnotetext{
${ }^{2}$ This also implies that we ignore any global stock effect in the representation of households' preferences, but this is because of the nature of the aggregate externality considered here. Following Weitzman (2007) in p. 713, the damage associated with the global stock effect is not modeled entering the instantaneous utility function as a direct argument, but as changing parameters of the intertemporal utility function. Nevertheless, an important stream of literature considers that welfare depends on the stock of pollution rather than on the current flow (see Huang and Cai, 1994, Mohtadi, 1996, or Byrne, 1997). However, if the flow of pollution is increasing with production, then capital accumulation that increases future output also increases future flows of pollution. All in all, we have now a general consensus in the literature (Gradus and Smulders, 1993, or Reis, 2001) according to which, if we consider the stock of pollution as an argument in the utility function, we will obtain the same fundamental long-run results but at the cost of a more complex analysis. Only transitional dynamics would change significantly if pollution stock is directly involved in preferences. In any case, our interest here is in the long-run dynamics.
} 
To ensure that such a path may exist in this model we assume that the particular instantaneous utility function is multiplicatively separable and of the CIES form

$$
U(c(t), z(t))=\frac{c(t)^{1-\Phi}}{1-\Phi}(1-z(t))^{\alpha(1-\Phi)} .
$$

In this function, the parameter $\alpha\left(\equiv \frac{U_{1-z}}{U_{c}} \frac{1-z}{c}=-\frac{U_{z}}{U_{c}} \frac{1-z}{c}\right)$ represents the relative weight of environmental care in utility and is assumed to be positive and lower than one, $0<\alpha<1$. Moreover, the inverse of the constant intertemporal elasticity of substitution ${ }^{3}$ is allowed to take values above or below unity, $0<\Phi \lessgtr 1$. The previous utility function fulfills all the above mentioned assumptions concerning first and second derivatives. The strict concavity assumption requires as sufficient condition that the determinant of the Hessian matrix be positive, which implies the additional parameter constraint $\Phi>\frac{\alpha}{1+\alpha} .4$

\section{The optimal sustainable growth problem}

In the optimal sustainable growth problem, the benevolent planner has to consider, on one hand, the true social costs and benefits from pollution abatement and, on the other, he has to take into account the global negative effect from an eventual ecological catastrophe associated with the accumulated stock of pollutants, that is the existence of a pollution ceiling. In this setting, we define social welfare (which is the objective function of the planner) as

$$
\begin{array}{lc}
W=\int_{t_{0}}^{+\infty} \frac{c^{1-\Phi}}{1-\Phi}(1-z)^{\alpha(1-\Phi)} N^{\lambda} e^{-\rho\left(t-t_{0}\right)} d t & \text { if } S(\tau) \leqslant S^{\max } \quad \forall \tau \\
W^{c}=\int_{t_{0}}^{t^{c}} \frac{c^{1-\Phi}}{1-\Phi}(1-z)^{\alpha(1-\Phi)} N^{\lambda} e^{-\rho\left(t-t_{0}\right)} d t & \text { if } S(\tau)>S^{\max } \quad \forall \tau>t^{c}
\end{array}
$$

where $t^{c}<+\infty$ represents the date the pollution ceiling is reached. Beyond $S^{\text {max }}$, it is implicitly assumed that welfare drops to zero. Parameter $\rho$ is the social rate of time preference or discount rate. Parameter $\lambda \in[0,1]$ contributes to specify preferences, which are represented using a Millian, an intermediate, or a Benthamite intertemporal utility function. In one extreme, when $\lambda=0$ (average utilitarianism), the central planner maximizes per capita utility (average utility of consumption per capita). In the other, when $\lambda=1$ (classical utilitarianism), central planner maximizes total utility (the addition across total population of utilities of per capita consumption).$^{5}$

\footnotetext{
${ }^{3}$ From now on, intertemporal elasticity of substitution (IES) will refer to the conventional IES coefficient given by $\frac{-U c}{c \cdot U_{c c}}=\frac{1}{\Phi}$, because the utility function (2.12) shows a long-run IES coefficient given by $\frac{1}{\Phi-\alpha(1-\Phi)}$.

${ }^{4}$ Environmental literature has long dealt with the sign of the second order cross derivative of the instantaneous utility function (Michel and Rotillon, 1995, or Mohtadi, 1996). From $U\left(c(t), \frac{\mathcal{B}(t)}{Y(t)}\right)$ we know that utility indirectly depends on the environmental quality, which increases with abatement effort scaled by economy's dimension. The latter can be measured by output as well as by capital stock, given the linear form of the production function. In the particular case of equation (2.12) we get $U_{c 2}=-U_{c z}=\alpha(1-\Phi) c^{-\Phi}(1-z)^{\alpha(1-\Phi)-1}$, which is negative (positive) as long as $\Phi$ is greater (smaller) than one. That is, as long as the intertemporal elasticity of substitution is smaller (greater) than one. Empirical evidence seems to corroborate the case of a low intertemporal elasticity of substitution and, hence, $U_{c 2}<0$. This implies that the marginal utility of consumption decreases as the environmental quality increases. Namely, consumption and pollution emissions are complements in terms of preferences. However, the model works exactly the same in the opposite case in which $U_{c 2}>0$.

${ }^{5}$ The literature differentiates between two types of altruism depending on the two parameters $\rho$ and $\lambda$. The first one is intertemporal altruism and depends on the discount rate applied to future population utility. The second one is intergenerational altruism and depends on the number of individuals which is taken into account each period. In particular, for representative and infinitely lived agent models, parameter $\lambda$ controls for the degree of altruism towards total population including future generations. When agents are (partially) selfish, $\lambda=0$, they care only about per capita utility (current and future), and the size of population has no direct effect on the intertemporal utility. Instead, when agents are (almost perfectly) altruistic, $\lambda=1$, they care not only about their own utility but also about that of their dynasties. In this case, the intertemporal utility function includes total population as a determinant, regardless of its value in the future. When $0<\lambda<1$ agents show an intermediate degree of intergenerational altruism.
} 
If the central planner is intended to maximizing social welfare, he can reach this target by maximizing $W$ subject to the constraint $S(t) \leqslant S^{\max }$. The corresponding dynamic optimization problem is thus formulated introducing an explicit constraint, the no-catastrophe condition as a pollution ceiling restriction, which implies that the central planner takes care ex-ante of trajectories leading to catastrophic states and optimally decides to avoid them by choosing the controls appropriately. A comprehensive account of all state and control constraints leads to list

$$
\begin{aligned}
& \text { (i) } z(t) \geqslant 0, \\
& \text { (ii) } 1-z(t) \geqslant 0, \\
& \text { (iii) } S(t) \leqslant S^{\max }
\end{aligned}
$$

which add to the usual dynamic and boundary constraints for $K$ and $S$. The third kind of constraint in (3.1) is a pure state constraint. It can be treated using a well-known textbook technique (see for example, Seierstad and Sydsaeter, 1987, Chap 5, Theorem 4). Concretely, writing the current stock in per capita and efficiency units, $\widetilde{s}(t)=\frac{S(t)}{A(t) N(t)}$, we get $S^{\max }-A(t) N(t) \widetilde{s}(t) \geqslant 0$, and given that $A(t) N(t) \widetilde{s}(t)$ is not allowed to exceed $S^{\text {max }}$, one can extract the following new condition when the state constraint is binding

$$
\begin{gathered}
\frac{d(A(t) N(t) \widetilde{s}(t))}{d t \quad}=A(t) N(t)(\widetilde{g}(1-z(t))-\delta(t) \widetilde{s}(t)) \leqslant 0 \\
\text { whenever } A(t) N(t) \widetilde{s}(t)=S^{\max },
\end{gathered}
$$

where we have made use of the equation representing the motion of the stock of pollutants in per capita and efficiency terms, i.e. $\dot{\widetilde{s}}(t)=\widetilde{g}(1-z(t))-(\delta(t)+x(t)+n(t)) \widetilde{s}(t){ }^{6}$

Hence, the planner's problem consists in choosing the sequence $\left\{c(t), z(t), t \geq t_{0}\right\}$ which, for a given positive social rate of discount $\rho>\bar{n}$, solve the optimization problem

$$
\max _{\{K, S, c, z\}} \int_{t_{0}}^{+\infty} \frac{c^{1-\Phi}}{1-\Phi}(1-z)^{\alpha(1-\Phi)} N^{\lambda} e^{-\rho\left(t-t_{0}\right)} d t
$$

s.t. $\quad(2.1),(2.2),(2.3),(2.5),(2.6),(2.7),(2.9),(2.11),(3.1)$ and (3.2),

$$
\text { given } A\left(t_{0}\right)=A_{0}>0, K\left(t_{0}\right)=K_{0}>0, N\left(t_{0}\right)=N_{0}>0 \text { and } S\left(t_{0}\right)=S_{0}>0 .
$$

Now we define variables $\widetilde{c}(t)=\frac{c(t)}{A(t)}$ and $\widetilde{k}(t)=\frac{K(t)}{A(t) N(t)}$ in per capita and efficiency terms. Then, the general dynamic optimization problem may be written as in the following current value (generalized) Hamiltonian

$$
\begin{gathered}
\underset{\{\widetilde{c}, z, q, \widetilde{k}, \mu, \widetilde{s}, \eta, \theta\}}{H^{c}}=\frac{\widetilde{c}^{1-\Phi}(1-z)^{\alpha(1-\Phi)}}{1-\Phi} A^{1-\Phi} N^{\lambda}+q\left(z \widetilde{k}^{\beta}-\widetilde{c}-(x+n) \widetilde{k}\right) \\
+\mu(\widetilde{g}(1-z)-(\delta+x+n) \widetilde{s})+\eta(1-z)-\theta A N(\widetilde{g}(1-z)-\delta \widetilde{s}) .
\end{gathered}
$$

\footnotetext{
${ }^{6}$ Recall that $G(1-z)$ is a strictly concave function representing the aggregate emissions flow, which depends positively on the dirtiness index $z$. Hence, the function $\widetilde{g}(1-z)$, which represents the efficiency per capita emissions flow, preserves the characterization attributed to the aggregate function: $\widetilde{g}^{\prime}<0, \lim _{x \rightarrow 0^{+}} \widetilde{g}^{\prime} \leq 0,-\infty<\lim _{x \rightarrow 1^{-}} \widetilde{g}^{\prime}<0, \widetilde{g}^{\prime \prime}<0, G^{M} \geqslant \widetilde{g}(0)=$ $\frac{G^{M}}{A(t) N(t)}>0$, and $\widetilde{g}(1)=0$. Moreover, $\widetilde{g}_{z}=-\widetilde{g}^{\prime}>0,0<\lim _{z \rightarrow 0^{+}} \widetilde{g}_{z}<+\infty, \lim _{z \rightarrow 1^{-}} \widetilde{g}_{z} \geq 0$, and $\widetilde{g}_{z z}=\widetilde{g}^{\prime \prime}<0$.
} 
Here, $q$ and $\mu$ are the co-states for $\widetilde{k}$ and $\widetilde{s}$ respectively, and $\eta$ and $\theta$ are Lagrangian multipliers associated with the constraint on the control-variable $z$ and the constraint on the state-variable $S$ respectively. Both $\eta$ and $\theta$ are dynamic multipliers because their corresponding constraints must be satisfied at every period $t$. Given that the control inequality constraint is linear, the first order necessary conditions arising from Pontryagin's principle and Kuhn-Tucker theorem are

$$
\begin{gathered}
q=\widetilde{c}^{-\Phi}(1-z)^{\alpha(1-\Phi)} A^{1-\Phi} N^{\lambda}, \\
q \widetilde{k}^{\beta}+\mu \widetilde{g}_{z}-\eta-\theta A N \widetilde{g}_{z}-\frac{\alpha \widetilde{c}^{1-\Phi}(1-z)^{\alpha(1-\Phi)}}{1-z} A^{1-\Phi} N^{\lambda} \leqslant 0, \\
z \geqslant 0, z\left(q \widetilde{k}^{\beta}+\mu \widetilde{g}_{z}-\eta-\theta A N \widetilde{g}_{z}-\frac{\alpha \widetilde{c}^{1-\Phi}(1-z)^{\alpha(1-\Phi)}}{1-z} A^{1-\Phi} N^{\lambda}\right)=0, \\
\dot{\widetilde{k}}=z \widetilde{k}^{\beta}-\widetilde{c}-(x+n) \widetilde{k}, \\
\dot{q}=(\rho+x+n) q-\beta z \widetilde{k}^{\beta-1} q, \\
\dot{\widetilde{s}}=\widetilde{g}(1-z)-(\delta+x+n) \widetilde{s}, \\
\dot{\mu}=(\rho+\delta+x+n) \mu-\delta A N \theta, \\
1-z \geqslant 0, \eta \geqslant 0, \eta(1-z)=0, \\
\widetilde{g}(1-z)-\delta \widetilde{s} \leqslant 0, \theta \geqslant 0, \theta(\widetilde{g}(1-z)-\delta \widetilde{s})=0,
\end{gathered}
$$

where

$$
\begin{aligned}
A(t) & =\frac{A_{0}}{\frac{A_{0}}{\bar{A}}+\left(1-\frac{A_{0}}{\bar{A}}\right) \exp \left(-\bar{x}\left(t-t_{0}\right)\right)}, \\
x(t) & =\frac{\bar{x}\left(\bar{A}-A_{0}\right)}{A_{0} \exp \left(\bar{x}\left(t-t_{0}\right)\right)+\left(\bar{A}-A_{0}\right)}, \\
N(t) & =\frac{N_{0}}{\frac{N_{0}}{\bar{N}}+\left(1-\frac{N_{0}}{\bar{N}}\right) \exp \left(-\bar{n}\left(t-t_{0}\right)\right)}, \\
n(t) & =\frac{\bar{n}\left(\bar{N}-N_{0}\right)}{N_{0} \exp \left(\bar{n}\left(t-t_{0}\right)\right)+\left(\bar{N}-N_{0}\right)} .
\end{aligned}
$$

To make clear that (3.8), (3.9), and (3.10) only apply when $A(t) N(t) \widetilde{s}(t)=S^{\text {max }}$ we append the complementary-slackness condition and the restriction on the way $\theta$ changes over time (see again Seierstad and Sydsaeter, 1987, Chap 5, Theorem 4)

$$
S \leqslant S^{\max }, \theta\left(S-S^{\max }\right)=0, \dot{\theta} \leqslant 0 \quad\left(=0 \text { when } S<S^{\max }\right) .
$$

Finally, we also need the initial conditions $\widetilde{k}_{0}$ and $\widetilde{s}_{0}$, and the transversality conditions

$$
\begin{aligned}
& \lim _{t \rightarrow+\infty} e^{-\rho\left(t-t_{0}\right)} q \widetilde{k}=0, \\
& \lim _{t \rightarrow+\infty} e^{-\rho\left(t-t_{0}\right)} \mu \widetilde{s}=0 .
\end{aligned}
$$


These necessary conditions are also sufficient for a maximum because the Hamiltonian function satisfies the required concavity conditions.

Using the particular functions (2.8) and (2.10) we get the following explicit relationships which will be useful later

$$
\begin{gathered}
\widetilde{g}(1-z)=\frac{G^{M}}{A N}\left(1-(1-z)^{2}\right) \\
\widetilde{g}_{z}=\frac{2 G^{M}}{A N}(1-z) \\
\delta(t)=\frac{\left(\delta_{0}-\bar{\delta}\right) \bar{N}}{N_{0} \exp \left(\bar{n}\left(t-t_{0}\right)\right)+\left(\bar{N}-N_{0}\right)}+\bar{\delta}
\end{gathered}
$$

As one can see, the problem is analytically highly complex, it gives rise to the dynamic system (3.3)(3.17) which is clearly intractable in many respects. Indeed, the system is not only nonlinear and simultaneous but it is also non-autonomous, given the assumed technological and demographic laws of motion. This said, we show hereafter that a partial analytical characterization is still possible although far nontrivial. First of all, we shall start by characterizing (as finely as we can) the non-binding regime, that is the case where the trajectories stay below the pollution ceiling permanently (Section 4). We then do the same when the economy lies on the binding regime (Section 5). In both cases, we try to characterize the long term dynamics in closed-form. It's worth pointing out here that if the exogenous variables, $A(t)$ and $N(t)$ were exponential functions, writing variables in efficiency units (that's dividing them by the product $A(t) N(t)$ ) would render the considered dynamic system autonomous with constant steady state solutions. In our benchmark model, both technology and population follow logistic curves, and such a transformation does not make the system autonomous. Constant steady state solutions only exist asymptotically. This will be quite apparent throughout the rest of the paper.

\section{The non-binding regime}

When the static constraints $(3.1)$ of the previous optimization problem are non-binding, $0<z(t)<1$ and $S(t)<S^{\text {max }}$, then $\theta=0, \dot{\theta}=0$ and $\eta=0$

$$
\begin{gathered}
q=\widetilde{c}^{-\Phi}(1-z)^{\alpha(1-\Phi)} A^{1-\Phi} N^{\lambda} \\
q \widetilde{k}^{\beta}+\mu \frac{2 G^{M}}{A N}(1-z)-\frac{\alpha \widetilde{c}^{1-\Phi}(1-z)^{\alpha(1-\Phi)}}{1-z} A^{1-\Phi} N^{\lambda}=0 \\
\dot{\widetilde{k}}=z \widetilde{k}^{\beta}-\widetilde{c}-(x+n) \widetilde{k} \\
\dot{q}=(\rho+x+n) q-\beta z \widetilde{k}^{\beta-1} q, \\
\dot{\widetilde{s}}=\frac{G^{M}}{A N}\left(1-(1-z)^{2}\right)-(\delta+x+n) \widetilde{s} \\
\dot{\mu}=(\rho+\delta+x+n) \mu
\end{gathered}
$$


Together with the initial conditions $\widetilde{k}_{0}$ and $\widetilde{s}_{0}$, and the transversality conditions

$$
\begin{gathered}
\lim _{t \rightarrow+\infty} e^{-\rho\left(t-t_{0}\right)} q(t) \widetilde{k}(t)=0, \\
\lim _{t \rightarrow+\infty} e^{-\rho\left(t-t_{0}\right)} \mu(t) \frac{S(t)}{A(t) N(t)}=0 .
\end{gathered}
$$

Moreover, there is also the exogenous dynamics given by trajectories (3.11), (3.12), (3.13), (3.14), and (3.18).

Next, after some preliminary algebraic manipulations, we characterize the long term equilibria associated with this regime (assuming that it is permanent) and we end up identifying a sufficient condition under which this regime cannot be optimally permanent, which we will validate moving to the second regime, the binding regime.

First of all, we take equation (4.2) getting as solution

$$
\mu(t)=\mu\left(t_{0}\right) e^{\int_{t_{0}}^{t}(\rho+\delta+x+n) d \tau}=\mu\left(t_{0}\right) e^{(\rho+\bar{\delta})\left(t-t_{0}\right)}\left(\frac{N(t)}{N_{0}}\right)^{\frac{\delta_{0}-\bar{\delta}}{\bar{n}\left(1-\frac{N_{0}}{\bar{N}}\right)}} \frac{A(t)}{A_{0}} \frac{N(t)}{N_{0}} .
$$

Second, we integrate (4.1) and obtain the expression

$$
S(t)=\left[e^{-\bar{\delta}\left(t-t_{0}\right)}\left(\frac{N(t)}{N_{0}}\right)^{\frac{-\left(\delta_{0}-\bar{\delta}\right)}{\bar{n}\left(1-\frac{N_{0}}{\bar{N}}\right)}}\right]\left[S_{0}+\int_{t_{0}}^{t} G^{M} z(\tau)(2-z(\tau))\left(\frac{N(\tau)}{N_{0}}\right)^{\frac{\delta_{0}-\bar{\delta}}{\bar{n}\left(1-\frac{N_{0}}{\bar{N}}\right)}} e^{\bar{\delta}\left(\tau-t_{0}\right)} d \tau\right] .
$$

Finally, substituting both expressions into the transversality condition (4.3) it results that

$$
\frac{\mu\left(t_{0}\right)}{A_{0} N_{0}}\left\{S_{0}+\int_{t_{0}}^{\infty} G^{M} z(\tau)(2-z(\tau))\left(\frac{N(\tau)}{N_{0}}\right)^{\frac{\delta_{0}-\bar{\delta}}{\bar{n}\left(1-\frac{N_{0}}{\bar{N}}\right)}} e^{\bar{\delta}\left(\tau-t_{0}\right)} d \tau\right\}=0 .
$$

And this condition holds if, and only if, $\mu\left(t_{0}\right)=0$ because the integral on the r.h.s. cannot be negative. Consequently, we may conclude that, $\forall t \geq t_{0}$,

$$
\mu(t)=0
$$

This result implies that even if the central planner internalizes all the pollution-based externalities, he optimally assigns zero value to the social shadow price of the accumulated stock of pollutants.

Our equations can be further reduced to

$$
\begin{gathered}
\widetilde{c}=\alpha^{\frac{\alpha(1-\Phi)}{\Phi-\alpha(1-\Phi)}}\left(A^{1-\Phi} N^{\lambda}\right)^{\frac{1}{\Phi-\alpha(1-\Phi)}} q^{\frac{-1}{\Phi-\alpha(1-\Phi)} \widetilde{k}^{\frac{-\beta \alpha(1-\Phi)}{\Phi-\alpha(1-\Phi)}}} \\
z=1-\alpha^{\frac{\Phi}{\Phi-\alpha(1-\Phi)}}\left(A^{1-\Phi} N^{\lambda}\right)^{\frac{1}{\Phi-\alpha(1-\Phi)}} q^{\frac{-1}{\Phi-\alpha(1-\Phi)} \widetilde{k}^{\frac{-\beta \Phi}{\Phi-\alpha(1-\Phi)}}}, \\
\dot{\widetilde{k}}=-(x+n) \widetilde{k}+\widetilde{k}^{\beta}-\left(\alpha^{\frac{\Phi}{\Phi-\alpha(1-\Phi)}}+\alpha^{\frac{\alpha(1-\Phi)}{\Phi-\alpha(1-\Phi)}}\right)\left(A^{1-\Phi} N^{\lambda}\right)^{\frac{1}{\Phi-\alpha(1-\Phi)}} q^{\frac{-1}{\Phi-\alpha(1-\Phi)}} \widetilde{k}^{\frac{-\beta \alpha(1-\Phi)}{\Phi-\alpha(1-\Phi)}}, \\
\dot{q}=(\rho+x+n) q-\beta q \widetilde{k}^{\beta-1}+\beta \alpha^{\frac{\Phi}{\Phi-\alpha(1-\Phi)}}\left(A^{1-\Phi} N^{\lambda}\right)^{\frac{1}{\Phi-\alpha(1-\Phi)}} q^{\frac{-1}{\Phi-\alpha(1-\Phi)}+1} \widetilde{k}^{\frac{-\beta \alpha(1-\Phi)}{\Phi-\alpha(1-\Phi)}-1},
\end{gathered}
$$




$$
\begin{gathered}
\lim _{t \rightarrow+\infty} e^{-\rho\left(t-t_{0}\right)} q(t) \widetilde{k}(t)=0, \\
\dot{\widetilde{s}}=\frac{G^{M}}{A N}\left(1-(1-z)^{2}\right)-(\delta+x+n) \widetilde{s}, \\
S(t)<S^{\max },
\end{gathered}
$$

together with (3.11)-(3.14) and (3.18). We are now able to investigate the existence of long term equilibria assuming that the non-binding regime is permanent.

\subsection{The stationary equilibria}

Because of the exogenous logistic dynamics for technology and demography, the dynamic systems depicted above are nonlinear non-autonomous despite variables are written in efficiency units. Accordingly, we cannot define stationary equilibria as traditionally (that's by zeroing the time derivatives of the variables written in efficiency units). We shall proceed heuristically to uncover the long-term behaviour of the solution paths taking into account that both technology and population size converge to finite values when $t$ tends to infinity. Suppose that $A$ and $N$ are equal to their steady state values, $A=\bar{A}, N=\bar{N}$ and $n=x=0$. Then, applying to equations (4.5)-(4.6), with $A$ and $N$ fixed to the latter values, the stationarity conditions $\dot{\widetilde{k}}=\dot{q}=0$

$$
\begin{gathered}
0=\widetilde{k}^{* \beta}-\left(\alpha^{\frac{\Phi}{\Phi-\alpha(1-\Phi)}}+\alpha^{\frac{\alpha(1-\Phi)}{\Phi-\alpha(1-\Phi)}}\right)\left(\bar{A}^{1-\Phi} \bar{N}^{\lambda}\right)^{\frac{1}{\Phi-\alpha(1-\Phi)}} q^{* \frac{-1}{\Phi-\alpha(1-\Phi)}} \widetilde{k}^{* \frac{-\beta \alpha(1-\Phi)}{\Phi-\alpha(1-\Phi)}}, \\
0=\rho q^{*}-\beta q^{*} \widetilde{k}^{* \beta-1}+\beta \alpha^{\frac{\Phi}{\Phi-\alpha(1-\Phi)}}\left(\bar{A}^{1-\Phi} \bar{N}^{\lambda}\right)^{\frac{1}{\Phi-\alpha(1-\Phi)}} q^{* \frac{-1}{\Phi-\alpha(1-\Phi)}}+1 \widetilde{k}^{* \frac{-\beta \alpha(1-\Phi)}{\Phi-\alpha(1-\Phi)}-1} .
\end{gathered}
$$

one gets

$$
\widetilde{k}_{\infty}=\left(\frac{\beta}{(1+\alpha) \rho}\right)^{\frac{1}{1-\beta}}
$$

and

$$
q_{\infty}^{*}=\left(\alpha^{\frac{\Phi}{\Phi-\alpha(1-\Phi)}}+\alpha^{\frac{\alpha(1-\Phi)}{\Phi-\alpha(1-\Phi)}}\right)^{\Phi-\alpha(1-\Phi)} \bar{A}^{1-\Phi} \bar{N}^{\lambda}\left(\frac{(1+\alpha) \rho}{\beta}\right)^{\frac{\beta \Phi}{1-\beta}} .
$$

Heuristically, one can also visualize the "long-term trajectories" of both variables when technology and demography approach their steady state values. These trajectories will be most useful in the numerical solution of the model. It's enough to apply again to equations (4.5)-(4.6), with $A$ and $N$ tending to their limit values, the stationarity conditions $\dot{\widetilde{k}}=\dot{q}=0$. One gets

$$
\widetilde{k}^{*}(t)=\left(\frac{\beta}{(1+\alpha) \rho+(1+\alpha(1-\beta))(x(t)+n(t))}\right)^{\frac{1}{1-\beta}},
$$

and

$$
q^{*}(t)=\left(\alpha^{\frac{\Phi}{\Phi-\alpha(1-\Phi)}}+\alpha^{\frac{\alpha(1-\Phi)}{\Phi-\alpha(1-\Phi)}}\right)^{\Phi-\alpha(1-\Phi)} A(t)^{1-\Phi} N(t)^{\lambda}
$$




$$
\cdot \frac{1}{\beta^{\frac{\beta \Phi}{1-\beta}}} \frac{((1+\alpha) \rho+(1+\alpha(1-\beta))(x(t)+n(t)))^{\frac{\Phi-(1-\beta) \alpha(1-\Phi)}{1-\beta}}}{((1+\alpha) \rho+(1+\alpha)(1-\beta)(x(t)+n(t)))^{\Phi-\alpha(1-\Phi)}} .
$$

One can easily check that:

$$
\widetilde{k}_{\infty}^{*} \equiv \lim _{t \rightarrow+\infty} \widetilde{k}^{*}(t)
$$

and

$$
q_{\infty}^{*} \equiv \lim _{t \rightarrow+\infty} q^{*}(t)
$$

We can proceed in the same heuristic way to characterize the "long-term" trajectories of the controls:

$$
\begin{gathered}
\widetilde{c}^{*}(t)=\beta^{\frac{\beta}{1-\beta}} \frac{\rho+(1-\beta)(x(t)+n(t))}{((1+\alpha) \rho+(1+\alpha(1-\beta))(x(t)+n(t)))^{\frac{1}{1-\beta}}}, \\
\widetilde{c}_{\infty}^{*} \equiv \lim _{t \rightarrow+\infty} \widetilde{c}^{*}(t)=\frac{1}{1+\alpha}\left(\frac{\beta}{(1+\alpha) \rho}\right)^{\frac{\beta}{1-\beta}}, \\
0<z^{*}(t)=\frac{\rho+x(t)+n(t)}{(1+\alpha) \rho+(1+\alpha(1-\beta))(x(t)+n(t))}<1, \\
0<z_{\infty}^{*} \equiv \lim _{t \rightarrow+\infty} z^{*}(t)=\frac{1}{1+\alpha}<1 .
\end{gathered}
$$

From the above expressions, we also get the heuristic long-term trajectories for some additional variables

$$
\begin{gathered}
\frac{\widetilde{c}^{*}(t)}{\widetilde{k}^{*}(t)}=\frac{\rho+(1-\beta)(x(t)+n(t))}{\beta}, \\
K^{*}(t)=A(t) N(t)\left(\frac{\lim _{t \rightarrow+\infty} \frac{\widetilde{c}^{*}(t)}{\widetilde{k}^{*}(t)}=\frac{\rho}{\beta},}{(1+\alpha) \rho+(1+\alpha(1-\beta))(x(t)+n(t))}\right)^{\frac{1}{1-\beta}}, \\
K_{\infty}^{*} \equiv \lim _{t \rightarrow+\infty} K^{*}(t)=\bar{A} \bar{N}\left(\frac{\beta}{(1+\alpha) \rho}\right)^{\frac{1}{1-\beta}}, \\
c_{\infty}^{*} \equiv \lim _{t \rightarrow+\infty} c^{*}(t)=\frac{\bar{A}}{1+\alpha}\left(\frac{\beta}{(1+\alpha) \rho}\right)^{\frac{\beta}{1-\beta}} .
\end{gathered}
$$

Finally, using (4.4), one can do the same for the aggregate stock of pollutants $S$ 


$$
\begin{gathered}
S^{*}(t)=\left(\frac{N_{0}}{\bar{N}}+\left(1-\frac{N_{0}}{\bar{N}}\right) \exp \left(-\bar{n}\left(t-t_{0}\right)\right)\right)^{\frac{\delta_{0}-\bar{\delta}}{\bar{n}\left(1-\frac{N_{0}}{\bar{N}}\right)}} \\
{\left[S_{0} e^{-\bar{\delta}\left(t-t_{0}\right)}+\int_{t_{0}}^{t} \frac{G^{M} z^{*}(\tau)\left(2-z^{*}(\tau)\right) e^{-\bar{\delta}(t-\tau)}}{\left.\left(\frac{N_{0}}{\bar{N}}+\left(1-\frac{N_{0}}{\bar{N}}\right) \exp \left(-\bar{n}\left(\tau-t_{0}\right)\right)\right)^{\frac{\delta_{0}-\bar{s}}{\bar{n}\left(1-\frac{N_{0}}{\bar{N}}\right)}} d \tau\right]}\right]}
\end{gathered}
$$

where

$$
0<z^{*}(t)\left(2-z^{*}(t)\right)=1-\left(\frac{\alpha \rho+\alpha(1-\beta)(x(t)+n(t))}{(1+\alpha) \rho+(1+\alpha(1-\beta))(x(t)+n(t))}\right)^{2}<1 .
$$

\subsection{The ecological catastrophe}

It's now possible to derive conditions under which the non-binding regime cannot be permanent, implying the optimal switch to a regime where pollution stock is equal to its ceiling. Indeed, given that $x(t)$ and $n(t)$ are both monotonous decreasing functions, and that $\frac{\partial z^{*}}{\partial(x+n)}>0$, and provided that $\frac{\partial\left[z^{*}\left(2-z^{*}\right)\right]}{\partial z^{*}}>$ $0 \forall 0<z^{*}<1$, one may conclude that

$$
\begin{gathered}
S^{*}(t) \geq S^{m}(t) \equiv\left(\frac{N_{0}}{\bar{N}}+\left(1-\frac{N_{0}}{\bar{N}}\right) \exp \left(-\bar{n}\left(t-t_{0}\right)\right)\right)^{\frac{\delta_{0}-\bar{\delta}}{\bar{n}\left(1-\frac{N_{0}}{\bar{N}}\right)}} \\
{\left[S_{0} e^{-\bar{\delta}\left(t-t_{0}\right)}+G^{M} \frac{1+2 \alpha}{(1+\alpha)^{2}} \int_{t_{0}}^{t} \frac{e^{-\bar{\delta}(t-\tau)}}{\left(\frac{N_{0}}{\bar{N}}+\left(1-\frac{N_{0}}{\bar{N}}\right) \exp \left(-\bar{n}\left(\tau-t_{0}\right)\right)\right)^{\frac{\delta_{0}-\bar{\delta}}{\bar{n}\left(1-\frac{N_{0}}{\bar{N}}\right)}}} d \tau\right] .}
\end{gathered}
$$

The previous integral has an explicit solution in terms of the Gaussian Hypergeometric function

$$
\begin{gathered}
\int_{t_{0}}^{t} \frac{e^{-\bar{\delta}(t-\tau)}}{\left(\frac{N_{0}}{\bar{N}}+\left(1-\frac{N_{0}}{\bar{N}}\right) \exp \left(-\bar{n}\left(\tau-t_{0}\right)\right)\right)^{\frac{\delta_{0}-\bar{\delta}}{\bar{n}\left(1-\frac{N_{0}}{\bar{N}}\right)}}} d \tau \\
=\frac{1}{\bar{\delta}}\left(\frac{1+\left(\frac{\bar{N}}{N_{0}}-1\right) \exp \left(-\bar{n}\left(t-t_{0}\right)\right)}{\frac{N_{0}}{\bar{N}}+\left(1-\frac{N_{0}}{\bar{N}}\right) \exp \left(-\bar{n}\left(t-t_{0}\right)\right)}\right)^{\frac{\delta_{0}-\bar{\delta}}{\bar{n}\left(1-\frac{N_{0}}{\bar{N}}\right)}}{ }_{2} F_{1}\left(a, b, 1+a ; d \exp \left(-\bar{n}\left(t-t_{0}\right)\right)\right) \\
-\frac{e^{-\bar{\delta}\left(t-t_{0}\right)}}{\bar{\delta}}\left(\frac{\bar{N}}{N_{0}}\right)^{\frac{\delta_{0}-\bar{\delta}}{\bar{n}\left(1-\frac{N_{0}}{\bar{N}}\right)}}{ }_{2} F_{1}(a, b, 1+a ; d),
\end{gathered}
$$

where $a=-\frac{\bar{\delta}}{\bar{n}}, b=\frac{\delta_{0}-\bar{\delta}}{\bar{n}\left(1-\frac{N_{0}}{\bar{N}}\right)}$, and $d=\left(1-\frac{\bar{N}}{N_{0}}\right)$.

This solution converges to 


$$
\lim _{t \rightarrow+\infty} \int_{t_{0}}^{t} \frac{e^{-\bar{\delta}(t-\tau)}}{\left(\frac{N_{0}}{\bar{N}}+\left(1-\frac{N_{0}}{\bar{N}}\right) \exp \left(-\bar{n}\left(\tau-t_{0}\right)\right)\right)^{\frac{\delta_{0}-\bar{\delta}}{\bar{n}\left(1-\frac{N_{0}}{\bar{N}}\right)}}} d \tau=\frac{1}{\overline{\bar{\delta}}}\left(\frac{\bar{N}}{N_{0}}\right)^{\frac{\delta_{0}-\bar{\delta}}{\bar{n}\left(1-\frac{N_{0}}{\bar{N}}\right)}}{ }_{2} F_{1}(a, b, 1+a ; 0),
$$

where ${ }_{2} F_{1}(a, b, 1+a ; 0)=1$. Consequently, we get

$$
S_{\infty}^{*} \equiv \lim _{t \rightarrow+\infty} S^{*}(t)=\lim _{t \rightarrow+\infty} S^{m}(t)=\frac{G^{M}}{\bar{\delta}} \frac{1+2 \alpha}{(1+\alpha)^{2}} .
$$

Then, a sufficient condition for the catastrophe, $S(t)>S^{\max }$, is

$$
G^{M} \frac{1+2 \alpha}{(1+\alpha)^{2}}>\bar{\delta} S^{\max }
$$

where $0.75<\frac{1+2 \alpha}{(1+\alpha)^{2}}<1$ because $1>\alpha>0$.

Recall that $\frac{1+2 \alpha}{(1+\alpha)^{2}}=z_{\infty}^{*}\left(2-z_{\infty}^{*}\right)$. So condition (4.12) is straightforward. In particular, a catastrophe is more likely to occur if (i) the pollution ceiling is low enough, (ii) if the asymptotic regeneration rate (the minimal value of this rate) is low enough, and (iii) if the emissions parameter $G^{M}$ is large enough. It is interesting to visualize the potential demographic connections in this picture. First of all, and though we have been agnostic in this respect so far, the asymptotic regeneration rate $\bar{\delta}$ may depend on maximal population $\bar{N}$ : naturally enough, and though this rate depends on many other potential factors as argued before, it is likely to be a decreasing function of $\bar{N}$. As such a larger population in the long-run favors ecological catastrophes. Second, when taking the model to the data (see Section 7 ), $G^{M}$ is captured by emissions indicators, and more precisely by emissions to GDP indicators. Therefore, a larger $\bar{N}$ would also increase the likelihood of a catastrophe. These scenarios are evaluated using UN projections in Section 7.

\section{The case where the pollution ceiling is reached}

If the sufficient condition identified just above holds, then pollution ceiling is reached and another regime (with binding state constraint) will set it. We shall characterize it hereafter. It is worth pointing out at this stage that in contrast to alternative optimal growth models with pollution ceiling (Amigues and Moreaux, 2013, includes another control as the extraction rate of nonrenewable resources but it could be investment in a backstop technology), which deliver optimal routes to leave the binding regime after a while, no such a route is possible here. Indeed, when the ceiling is reached the economy stays in this regime forever. We shall give hereafter some details on how this works.

Consider that we find $S\left(t^{c}\right)=S^{\max }$ for some $+\infty>t^{c}>t_{0}$. This implies that the absolute limit to growth, i.e. the ecological and economic catastrophe, appears as a binding constraint in finite time. In what follows we pay special attention to this case and we study the first order conditions at that moment and thereafter. The way the central planner can avoid the catastrophic at the lower cost in welfare terms is by keeping $S$ constant,

$$
S(t)=S\left(t^{c}\right)=S^{\max } \forall t \geqslant t^{c} .
$$


That is, $\dot{S}(t)=0 \quad \forall t \geqslant t^{c}$. Hence, $G\left(1-z\left(t^{c}\right)\right)=\delta\left(t^{c}\right) S^{*}\left(t^{c}\right)=\delta\left(t^{c}\right) S^{\text {max }}$ and, given the particular functions assumed in (2.8) and (2.10), we get

$$
z\left(t^{c}\right)^{2}-2 z\left(t^{c}\right)+\left(\frac{\left(\delta_{0}-\bar{\delta}\right) \bar{N}}{N_{0} \exp \left(\bar{n}\left(t^{c}-t_{0}\right)\right)+\left(\bar{N}-N_{0}\right)}+\bar{\delta}\right) \frac{S^{\max }}{G^{M}}=0
$$

Consequently, at $t^{c}$ the dirtiness index, a variable which admits jumps, will take the value

$$
0<z\left(t^{c}\right)=1-\sqrt{1-\left(\frac{\left(\delta_{0}-\bar{\delta}\right) \bar{N}}{N_{0} \exp \left(\bar{n}\left(t^{c}-t_{0}\right)\right)+\left(\bar{N}-N_{0}\right)} \frac{S^{\max }}{G^{M}}+\frac{\bar{\delta} S^{\max }}{G^{M}}\right)}<1,
$$

irrespective of its value in previous periods. This means that at $t^{c}$ the central planner may optimally decide, if necessary, a discrete and instantaneous change in the dirtiness index to the previous $z\left(t^{c}\right)$. Moreover, from (3.9) we get $\eta\left(t^{c}\right)=0$.

However, beyond $t^{c}$ population still grows according to (2.4) and, consequently, the rate of regeneration of the pollution stock diminishes according to (2.10). The process continues until population eventually reaches the carrying capacity $\bar{N}$, and the rate of natural regeneration eventually catches up with the minimum constant rate $\bar{\delta}$. During this period of time, to guarantee $\dot{S}(t)=0$ we need that $z(t)$ changes according to

$$
\begin{gathered}
0<z(t)=1-\sqrt{1-\left(\frac{\left(\delta\left(t^{c}\right)-\bar{\delta}\right) \bar{N}}{N\left(t^{c}\right) \exp \left(\bar{n}\left(t-t^{c}\right)\right)+\left(\bar{N}-N\left(t^{c}\right)\right)} \frac{S^{\max }}{G^{M}}+\frac{\bar{\delta} S^{\max }}{G^{M}}\right)}<1 \quad \forall t \geqslant t^{c}, \\
0<z_{\infty}=\lim _{t \rightarrow+\infty} z(t)=1-\sqrt{1-\frac{\bar{\delta} S^{\max }}{G^{M}}}<1 .
\end{gathered}
$$

Therefore $\eta(t)=0 \quad \forall t \geqslant t^{c}$. On other hand, condition (5.1) also implies $\widetilde{g}(1-z(t))=$ $\delta(t) \widetilde{s}(t) \quad \forall t \geqslant t^{c}$. Under (3.10) and (3.15), one gets $\theta(t) \geqslant 0$ and $\dot{\theta}(t) \leqslant 0 \quad \forall t \geqslant t^{c}$. This means that in case $\theta=0$ then $\dot{\theta}=0$, and in case $\theta>0$ then $\dot{\theta} \leqslant 0$. In other words, $\theta$ can be only nil, positive constant or positive decreasing forever.

From (3.7) and (3.10) the dynamic equation for $\widetilde{s}$ becomes $\stackrel{\grave{s}}{=}=-(x+n) \widetilde{s}$, from which we get

$$
\widetilde{s}(t)=\widetilde{s}\left(t^{c}\right) e^{-\int_{t^{c}}^{t}(x(\tau)+n(\tau)) d \tau} \quad \forall t \geqslant t^{c} .
$$

Moreover, the solution to the dynamic equation (3.8) is

$$
\mu(t)=e^{\int_{t^{c}}^{t}(\rho+\delta(\tau)+x(\tau)+n(\tau)) d \tau}\left[\mu\left(t^{c}\right)-\int_{t^{c}}^{t} \delta(\epsilon) A(\epsilon) N(\epsilon) \theta(\epsilon) e^{-\int_{t^{c}}^{\epsilon}(\rho+\delta(\alpha)+x(\alpha)+n(\alpha)) d \alpha} d \epsilon\right] \quad \forall t \geqslant t^{c} .
$$

The path for the shadow price $\mu$ couples to such of the stock $\widetilde{s}$, and the transversality condition (3.17) holds if, and only if,

$$
\mu\left(t^{c}\right)=A\left(t^{c}\right) N\left(t^{c}\right)^{\frac{\delta\left(t^{c}\right)-\bar{\delta}}{\bar{n}\left(1-\frac{N\left(t^{c}\right)}{\bar{N}}\right)}+1} \int_{t^{c}}^{+\infty} \delta(\tau) \theta(\tau) N(\tau)^{-\frac{\delta\left(t^{c}\right)-\bar{\delta}}{\bar{n}\left(1-\frac{N\left(t^{c}\right)}{\bar{N}}\right)}} e^{-(\rho+\bar{\delta})\left(\tau-t^{c}\right)} d \tau
$$


The previous integral is bounded. The integrand, which is the product of three non-negative nonincreasing terms times the enlarged discount factor, converges to zero. Consequently, we find that

$$
\begin{aligned}
& \mu(t)=\int_{t}^{+\infty} \delta(\tau) A(\tau) N(\tau) \theta(\tau) e^{-\int_{t}^{\tau}(\rho+\delta(r)+x(r)+n(r)) d r} d \tau \\
& =A(t) N(t)^{\frac{\delta(t)-\bar{\delta}}{\bar{n}\left(1-\frac{N(t)}{\bar{N}}\right)}+1} \int_{t}^{+\infty} \delta(\tau) \theta(\tau) N(\tau)^{-\frac{\delta(t)-\bar{\delta}}{\bar{n}\left(1-\frac{N(t)}{\bar{N}}\right)}} e^{-(\rho+\bar{\delta})(\tau-t)} d \tau>0 \quad \forall t \geqslant t^{c} .
\end{aligned}
$$

As expected, $\mu(t) \neq 0 \quad \forall t \geqslant t^{c}$ because all along this interval of time the pure state-space constraint is binding and, in the corresponding corner solution, the central planner optimally assigns a positive value to the social shadow price of the accumulated stock of pollutants.

The remaining first order conditions are

$$
\begin{gathered}
\widetilde{c}=\left(\frac{(1-z)^{\alpha(1-\Phi)} A^{1-\Phi} N^{\lambda}}{q}\right)^{\frac{1}{\Phi}}, \\
\dot{\widetilde{k}}=-(x+n) \widetilde{k}+z \widetilde{k}^{\beta}-\left((1-z)^{\alpha(1-\Phi)} A^{1-\Phi} N^{\lambda}\right)^{\frac{1}{\Phi}} q^{-\frac{1}{\Phi}}, \\
\dot{q}=(\rho+x+n) q-\beta z q \widetilde{k}^{\beta-1},
\end{gathered}
$$

where

$$
\begin{gathered}
A(t)=\frac{A\left(t^{c}\right)}{\frac{A\left(t^{c}\right)}{\bar{A}}+\left(1-\frac{A\left(t^{c}\right)}{\bar{A}}\right) \exp \left(-\bar{x}\left(t-t^{c}\right)\right)}, \\
x(t)=\frac{\bar{x}\left(1-\frac{A\left(t^{c}\right)}{\bar{A}}\right)}{\frac{A\left(t^{c}\right)}{\bar{A}} \exp \left(\bar{x}\left(t-t^{c}\right)\right)+\left(1-\frac{A\left(t^{c}\right)}{\bar{A}}\right)}, \\
N(t)=\frac{N\left(t^{c}\right)}{\frac{N\left(t^{c}\right)}{\bar{N}}+\left(1-\frac{N\left(t^{c}\right)}{\bar{N}}\right) \exp \left(-\bar{n}\left(t-t^{c}\right)\right)}, \\
n(t)=\frac{\bar{n}\left(1-\frac{N\left(t^{c}\right)}{\bar{N}}\right)}{\frac{N\left(t^{c}\right)}{\bar{N}} \exp \left(\bar{n}\left(t-t^{c}\right)\right)+\left(1-\frac{N\left(t^{c}\right)}{\bar{N}}\right)}, \\
\delta(t)=\left(\delta\left(t^{c}\right)-\bar{\delta}\right)\left(\frac{\delta\left(t^{c}\right)-\bar{\delta}}{\bar{N}-N(t)}\right)+\bar{\delta}=\frac{N\left(t^{c}\right)}{\bar{N}} \exp \left(\bar{n}\left(t-t^{c}\right)\right)+\left(1-\frac{N\left(t^{c}\right)}{\bar{N}}\right)
\end{gathered}
$$

Together with the initial condition $\widetilde{k}_{0}$ and the transversality condition

$$
\lim _{t \rightarrow+\infty} e^{-\rho\left(t-t^{c}\right)} q(t) \widetilde{k}(t)=0 .
$$




\subsection{Stationary equilibria when pollution ceiling is binding}

Using the same methodology as in Section 4.1, one can compute the "long-term" trajectories of the variables in the binding case. Based on equations (5.3)-(5.4), one gets:

$$
\begin{aligned}
& \widetilde{k}^{*}(t)=\left(\frac{\beta z(t)}{\rho+x(t)+n(t)}\right)^{\frac{1}{1-\beta}}, \\
& \widetilde{k}_{\infty}^{*} \equiv \lim _{t \rightarrow+\infty} \widetilde{k}^{*}(t)=\left(\frac{\beta z_{\infty}}{\rho}\right)^{\frac{1}{1-\beta}} \\
& q^{*}(t)=\frac{(1-z(t))^{\alpha(1-\Phi)} A(t)^{1-\Phi} N(t)^{\lambda}}{\left(z(t) \tilde{k}^{*}(t)^{\beta}-(x(t)+n(t)) \tilde{k}^{*}(t)\right)^{\Phi}}, \\
& q_{\infty}^{*} \equiv \lim _{t \rightarrow+\infty} q^{*}(t)=\frac{\left(1-z_{\infty}\right)^{\alpha(1-\Phi)} \bar{A}^{1-\Phi} \bar{N}^{\lambda}}{z_{\infty}^{\frac{\Phi}{1-\beta}}}\left(\frac{\rho}{\beta}\right)^{\frac{\beta \Phi}{1-\beta}} \\
& \widetilde{c}^{*}(t)=z(t) \widetilde{k}^{*}(t)^{\beta}-(x(t)+n(t)) \widetilde{k}^{*}(t), \\
& \widetilde{c}_{\infty}^{*} \equiv \lim _{t \rightarrow+\infty} \widetilde{c}^{*}(t)=z_{\infty}^{\frac{1}{1-\beta}}\left(\frac{\beta}{\rho}\right)^{\frac{\beta}{1-\beta}} \\
& \frac{\widetilde{c}^{*}(t)}{\widetilde{k}^{*}(t)}=\frac{\rho+(1-\beta)(x(t)+n(t))}{\beta}, \\
& \lim _{t \rightarrow+\infty} \frac{\widetilde{c}^{*}(t)}{\widetilde{k}^{*}(t)}=\frac{\rho}{\beta} \\
& K^{*}(t)=A(t) N(t)\left(\frac{\beta z(t)}{\rho+x(t)+n(t)}\right)^{\frac{1}{1-\beta}}, \\
& K_{\infty}^{*} \equiv \lim _{t \rightarrow+\infty} K^{*}(t)=\bar{A} \bar{N}\left(\frac{\beta z_{\infty}}{\rho}\right)^{\frac{1}{1-\beta}} \\
& c^{*}(t)=z(t) A(t) \widetilde{k}^{*}(t)^{\beta}-(x(t)+n(t)) A(t) \widetilde{k}^{*}(t),
\end{aligned}
$$

and

$$
c_{\infty}^{*} \equiv \lim _{t \rightarrow+\infty} c^{*}(t)=\bar{A} z_{\infty}^{\frac{1}{1-\beta}}\left(\frac{\beta}{\rho}\right)^{\frac{\beta}{1-\beta}}
$$

\subsection{Long-term environmental quality and consumption per capita in the nonbinding vs binding regime}

A useful exercise to understand the optimal solution and the associated optimal sustainable policy is to compare the long-run values for the dirtiness index and consumption per capita obtained for each regime (if they were permanent), both involved in welfare evaluations. A first important result that can be obtained is that the optimal policy operated when the ecological ceiling is reached is unambiguously 
stricter in the sense that abatement is clearly larger in the latter case yielding a better environmental quality (or in other words, a lower value for $z_{\infty}$ ). This can be proved readily. Assume (4.12):

$$
\frac{1+2 \alpha}{(1+\alpha)^{2}}>\frac{\bar{\delta} S^{\max }}{G^{M}},
$$

which implies that the economy optimally reaches the ceiling value for pollution in a finite time. Since, by (5.2), the asymptotic value of the dirtiness index is in this case

$$
z_{\infty}=1-\sqrt{1-\frac{\bar{\delta} S^{\max }}{G^{M}}}
$$

the following inequality holds

$$
z_{\infty}<1-\sqrt{1-\frac{1+2 \alpha}{(1+\alpha)^{2}}}
$$

which exactly yields

$$
z_{\infty}<\frac{1}{1+\alpha}
$$

Since any solution in the non-binding regime converges to $z_{\infty}^{*}=\frac{1}{1+\alpha}$ by (4.9), it follows as announced that when reaching the pollution ceiling, the environmental constraint requires a much stringer abatement policy, resulting in a clearly better environmental quality asymptotically (and better welfare everything equal elsewhere) than under any non-binding regime.

One could also compare the two regimes with respect to the asymptotic consumption per capita. According to (4.10), this value for the non-binding regime is equal to $\bar{A}\left(\frac{1}{1+\alpha}\right)^{\frac{1}{1-\beta}}\left(\frac{\beta}{\rho}\right)^{\frac{\beta}{1-\beta}}$. For the binding regime, the counterpart is given exactly by equation $(5.5)$ as equal to $\bar{A} z_{\infty}^{\frac{1}{1-\beta}}\left(\frac{\beta}{\rho}\right)^{\frac{\beta}{1-\beta}}$.

Since we have just proved above that $z_{\infty}<\frac{1}{1+\alpha}$, it follows that asymptotic consumption per capita in the binding regime is lower. This is the cost of fighting pollution at its ceiling level. This drop in consumption when the ceiling level is reached is quite common in the related literature of sustainable growth as documented in Amigues and Moreaux (2013).

\section{The case of exponential technological progress}

Now we consider a departure from the benchmark analysis conducted so far, and introduce the exponential law of motion for technology as it is typically the case in neoclassical growth theory. We keep population dynamics logistic. Concretely we assume

$$
A(t)=A_{0} \exp \left(\bar{x}\left(t-t_{0}\right)\right)
$$

where $\bar{x} \geq 0$ is the rate of technical progress. Function $\delta(\cdot)$ is defined again as in Section 2. Because technology is exogenously exponential, all variables (except $z$ ) will exhibit exponential growth asymptotically. We therefore detrend these variables, or in other terms, we rewrite them in efficiency units as we have been doing from Section 3. Following exactly the same steps as before, we are able to characterize the long-run dynamics and asymptotic values for the key variables in the non-binding regime. That is, to identify a sufficient condition for the emergence of the binding regime and then again deduce the corresponding long-run trajectories and the associated asymptotic long-run values for the relevant economic variables. Here we concentrate on the non-binding regime to unburden the exposition, the main objective of this section being comparison with the benchmark "logistic" model. Using the same heuristic methodology as 
in Section 4.1, we hereafter list the "long term" dynamics and associated asymptotic values for capital, consumption per capita (both in efficiency units), the dirtiness index and the stock of pollution:

$$
\begin{aligned}
& \widetilde{k}_{I I I}^{*}(t)=\left(\frac{\beta}{(1+\alpha) \rho+(\Phi-\alpha(\beta-\Phi)) \bar{x}+(1+\alpha(1-\beta)) n(t)}\right)^{\frac{1}{1-\beta}}, \\
& \lim _{t \rightarrow+\infty} \widetilde{k}_{I I I}^{*}(t)=\left(\frac{\beta}{(1+\alpha) \rho+(\Phi-\alpha(\beta-\Phi)) \bar{x}}\right)^{\frac{1}{1-\beta}} \\
& \widetilde{c}_{I I I}^{*}(t)=\frac{\beta^{\frac{\beta}{1-\beta}}}{(1+\alpha)} \frac{(1+\alpha) \rho+(\Phi-\alpha(\beta-\Phi)-\beta) \bar{x}+(1+\alpha)(1-\beta) n(t)}{((1+\alpha) \rho+(\Phi-\alpha(\beta-\Phi)) \bar{x}+(1+\alpha(1-\beta)) n(t))^{\frac{1}{1-\beta}}}, \\
& \lim _{t \rightarrow+\infty} \widetilde{c}_{I I I}^{*}(t)=\frac{\beta^{\frac{\beta}{1-\beta}}}{(1+\alpha)} \frac{(1+\alpha) \rho+(\Phi-\alpha(\beta-\Phi)-\beta) \bar{x}}{((1+\alpha) \rho+(\Phi-\alpha(\beta-\Phi)) \bar{x})^{\frac{1}{1-\beta}}}, \\
& z_{I I I}^{*}(t)=1-\frac{\alpha}{1+\alpha} \frac{(1+\alpha) \rho+(\Phi-\alpha(\beta-\Phi)-\beta) \bar{x}+(1+\alpha)(1-\beta) n(t)}{(1+\alpha) \rho+(\Phi-\alpha(\beta-\Phi)) \bar{x}+(1+\alpha(1-\beta)) n(t)}, \\
& \lim _{t \rightarrow+\infty} z_{I I I}^{*}(t)=1-\frac{\alpha}{1+\alpha} \frac{(1+\alpha) \rho+(\Phi-\alpha(\beta-\Phi)-\beta) \bar{x}}{(1+\alpha) \rho+(\Phi-\alpha(\beta-\Phi)) \bar{x}},
\end{aligned}
$$

and

$$
\begin{gathered}
S_{I I I}^{*}(t)=\left(\frac{N_{0}}{\bar{N}}+\left(1-\frac{N_{0}}{\bar{N}}\right) \exp \left(-\bar{n}\left(t-t_{0}\right)\right)\right)^{\frac{\delta_{0}-\bar{\delta}}{\bar{n}\left(1-\frac{N_{0}}{\bar{N}}\right)}} \\
\cdot\left[S_{0} e^{-\bar{\delta}\left(t-t_{0}\right)}+\int_{t_{0}}^{t} \frac{G^{M} z_{I I I}^{*}(\tau)\left(2-z_{I I I}^{*}(\tau)\right) e^{-\bar{\delta}(t-\tau)}}{\left(\frac{N_{0}}{\bar{N}}+\left(1-\frac{N_{0}}{\bar{N}}\right) \exp \left(-\bar{n}\left(\tau-t_{0}\right)\right)\right)^{\frac{\delta_{0}-\bar{\delta}}{\bar{n}\left(1-\frac{N_{0}}{\bar{N}}\right)}}} d \tau\right],
\end{gathered}
$$

where

$$
\begin{gathered}
z_{I I I}^{*}(t)\left(2-z_{I I I}^{*}(t)\right)=1-\left(\frac{\alpha}{1+\alpha} \frac{(1+\alpha) \rho+(\Phi-\alpha(\beta-\Phi)-\beta) \bar{x}+(1+\alpha)(1-\beta) n(t)}{(1+\alpha) \rho+(\Phi-\alpha(\beta-\Phi)) \bar{x}+(1+\alpha(1-\beta)) n(t)}\right)^{2}, \\
\lim _{t \rightarrow+\infty} S_{I I I}^{*}(t)=\frac{G^{M}}{\bar{\delta}}\left(1-\left(\frac{\alpha}{1+\alpha} \frac{(1+\alpha) \rho+(\Phi-\alpha(\beta-\Phi)-\beta) \bar{x}}{(1+\alpha) \rho+(\Phi-\alpha(\beta-\Phi)) \bar{x}}\right)^{2}\right) .
\end{gathered}
$$


We now compare the asymptotic values of these variables with their counterpart in Section 4, the optimal outcomes in the non-binding case when technological progress is logistic. ${ }^{7}$ Let us start with the dirtiness index given by equation (6.3). It is easy to check that we get the dirtiness index value corresponding to the logistic case given by equation (4.9) when $\bar{x}=0$. Since the latter is typically a small number, it is easy to visualize the impact of exponential growth by looking at the sign of the derivative of the dirtiness index given by (6.3) at $\bar{x}=0$. In a similar way this is also checked for (per capita and in efficiency units) physical capital and consumption, shown in equations (6.1), (4.7), (6.2), and (4.8), as well as for the (aggregate) stock of pollutants, shown in equations (6.4) and (4.11). The sign of their derivatives are

$$
\begin{gathered}
\left.\frac{\partial}{\partial \bar{x}}\left(\lim _{t \rightarrow+\infty} \widetilde{k}_{I I I}^{*}(t)\right)\right|_{\bar{x}=0}=-\frac{\beta^{\frac{1}{1-\beta}}(\Phi-\alpha(\beta-\Phi))}{(1-\beta)((1+\alpha) \rho)^{\frac{2-\beta}{1-\beta}}}<0 \\
\left.\frac{\partial}{\partial \bar{x}}\left(\lim _{t \rightarrow+\infty} \widetilde{c}_{I I I}^{*}(t)\right)\right|_{\bar{x}=0}=-\frac{(1-\beta+\Phi-\alpha(\beta-\Phi))}{(1+\alpha)(1-\beta)}\left(\frac{\beta}{(1+\alpha) \rho}\right)^{\frac{1}{1-\beta}}<0 \\
\left.\frac{\partial}{\partial \bar{x}}\left(\lim _{t \rightarrow+\infty} z_{I I I}^{*}(t)\right)\right|_{\bar{x}=0}=\frac{\rho \alpha \beta}{((1+\alpha) \rho)^{2}}>0 \\
\left.\frac{\partial}{\partial \bar{x}}\left(\lim _{t \rightarrow+\infty} S_{I I I}^{*}(t)\right)\right|_{\bar{x}=0}=\frac{G^{M}}{\bar{\delta}} \frac{2 \alpha^{2} \beta}{\rho(1+\alpha)^{3}}>0
\end{gathered}
$$

As one would expect, benefitting from an exogenous exponential output-augmenting technological progress gives the economy a wider margin to design optimal sustainable policies: in particular, the economy can afford in that case a less stringent optimal environmental policy and a larger stock of pollution than in the "logistic" economy. It should be also noted that though consumption and capital, both detrended, go down with exponential technical progress, these two variables exponentially grow in per capita terms in the long-run while per capita consumption and capital converge to constant levels in the logistic case. Ultimately, this exercise shows clearly to which extent the technological assumptions shape the sustainability problem under scrutiny: the latter is certainly much less critical when population grows logistically and technological progress evolves exponentially.

\section{Numerical experiments}

In this section we take the model to the data. Our benchmark economy, in which population and technical progress follow an exogenous logistic law of motion, is calibrated using the following specifications. We set $\phi=1.5$ and $\beta=0.4$, according to Caballé and Santos (1993) and Mulligan and Sala-i-Martin (1993). Then, we fix $\lambda=1$ to set that the planner maximizes the total utility of the society and population size is taking into account. The relative weight of environmental care in utility and the discount rate values have been chosen in order to have a consumption-output ratio close to 0.8 and a capital-output ratio around 3 ; we obtain $\rho=0.1$ and $\alpha=0.2$, which are values quite similar to standard values in the literature.

\footnotetext{
${ }^{7}$ As mentioned above, it is not difficult to do the same for the binding regime. One can easily identify as before a sufficient condition for an ecological catastrophe $S(t)>S^{\mathrm{max}}$, and proceed with optimal dynamics when the ceiling is reached. In this case

$$
G^{M}\left(1-\left(\frac{\alpha}{1+\alpha} \frac{(1+\alpha) \rho+(\Phi-\alpha(\beta-\Phi)-\beta) \bar{x}}{(1+\alpha) \rho+(\Phi-\alpha(\beta-\Phi)) \bar{x}}\right)^{2}\right)>\bar{\delta} S^{\max }
$$

where $0<\frac{(1+\alpha) \rho+(\Phi-\alpha(\beta-\Phi)-\beta) \bar{x}}{(1+\alpha) \rho+(\Phi-\alpha(\beta-\Phi)) \bar{x}}<1$ because $\rho-(1-\Phi) \bar{x}>0$.
} 
For the environmental parameters we set $\delta_{0}=0.014$ and, according to the literature, we fix $\bar{\delta}=0.005$, $S^{\max }=600$ and $G^{M}$ to be consistent with an emission-output ratio equal to 0.12 (see Boucekkine et al., 2013b; Hoel and Karp, 2002 and Nordhaus, 2008). Finally, we fix, for the technological parameters, $A(0)=1, \bar{A}=7, \bar{x}=0.02$ as reference values for all the numerical experiments.

Related to the demographic parameters we follow the United Nation population projections (UNPD, 2004). UN report shows three possible demographic scenarios depending on the evolution of the projected world population. We follow the estimated medium scenario from 2000 to 2050 as the baseline case; we normalize the population size in 2000 to $N(0)=1$ and, taking into account the population size in 2050, we calculate $\bar{N}=1.47$. Next, we use the population growth rate in 2000 to set $\bar{n}=0.04$ following equation (3.14). Under this parameterization $G^{M}=2.83$ and $S=551<S^{\max }$. Finally, we need to fix the initial conditions $k(0)$ and $S(0)$. In all our numerical experiments we assume that the economy is $40 \%$ below its long run value for both state variables (capital and pollution), and we check if the results are sensitive to initial conditions. Sensitivity analysis around these reference values reveal no qualitative change.

\subsection{Logistic and exponential technical progress}

To illustrate the analytical results founded in section 6 , we compare the solution paths of the relevant variables in two identical economies except for the law of motion of technological progress. In figures 1-2, dark lines represent the "logistic" economy and grey lines represent the "exponential" economy. Figure 1 shows the evolution of the pollution stock in both economies. Note that none of them reaches the pollution ceiling and they do converge to their respective long run values, $S=551$ (553) in the baseline case (exponential case). Since the small differences between both solution paths are hidden we also compare the same trajectories during the first 30 periods (see the right side of figure 1), showing that the exponential output-augmenting technological progress economy can optimally afford a larger stock of pollution than the "logistic economy". Consistently, the dirtiness index is higher in the "exponential" economy. Unlike the pollution stock trajectory, the rest of variables z, and detrended consumption show a higher convergence speed to its long-run value in the exponential output-augmenting technological progress economy (see figure 2). As we have mentioned in section 6, although detrended consumption is lower in the exponential output-augmenting technological progress economy along the transition to its long run level, it exponentially grows in the long run.
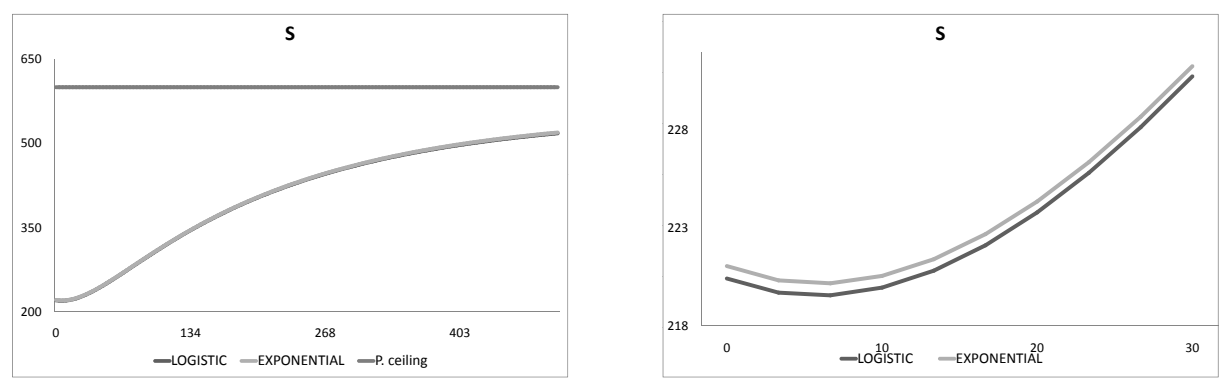

Figure 1. Pollution stock. 

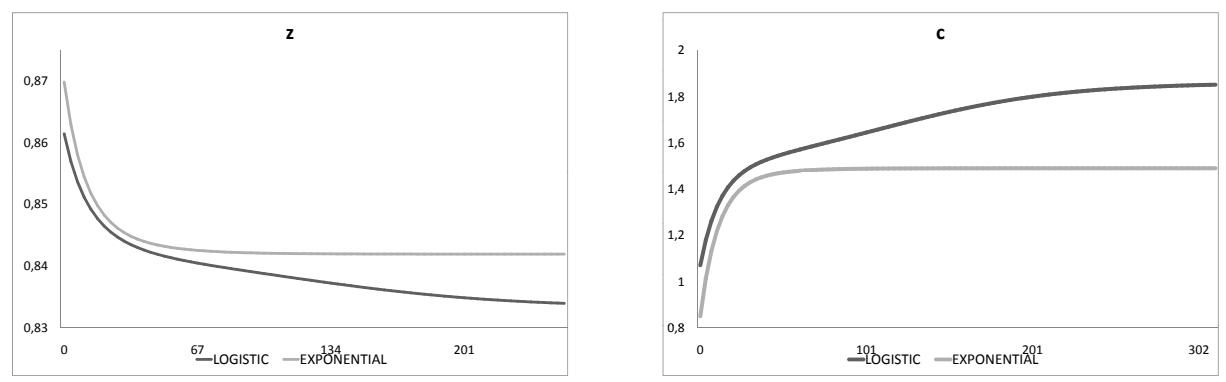

Figure 2. The dirtiness index and per capita consumption.

\subsection{The role of the altruism parameter}

Now we focus on the importance of the degree of intergenerational altruism assumed for economic agents in the outcomes of our benchmark economy. Recall that $\lambda$ contributes to determine agents preferences, which are represented using a Millian, an intermediate or a Benthamite intertemporal utility function. In sections 4 and 5 we show that the long-run trajectories of the relevant variables are independent of $\lambda$. Brida and Accinelli (2007) and Ferrara and Guerrini (2009) obtain a similar finding when analyzing the Ramsey model with a population logistic law of motion ${ }^{8}$ Since in exogenous growth models it seems that the altruism parameter plays no role along the balanced growth path, we consider interesting to explore its relevance on the short-run. In figures 3-4 the dark lines represent the altruistic case, that is $\lambda=1$, and the grey lines correspond to the selfish case, that is $\lambda=0$. Figure 3 (figure 4 ) shows the transitional dynamics of per capita consumption and the dirtiness index when technological progress is logistic (exponential).
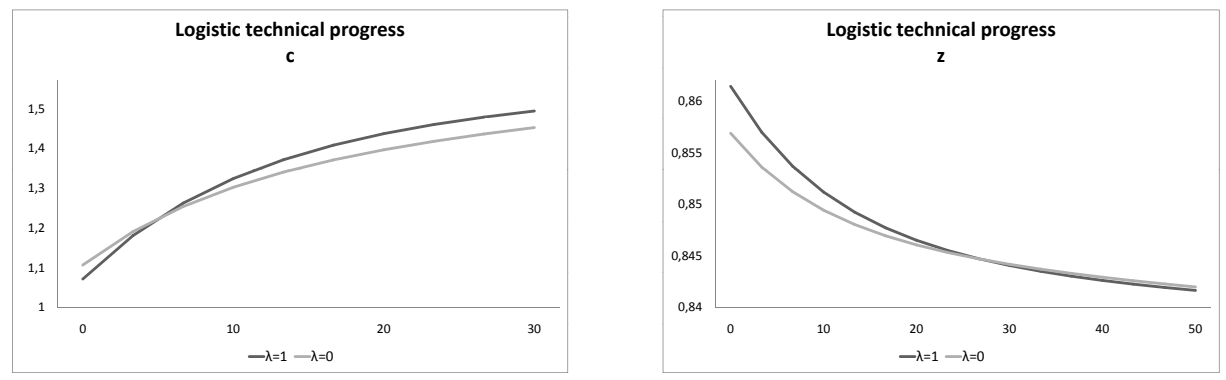

FiguRE 3. Consumption per capita and the dirtiness index when technical progress is logistic.

First, we concentrate on the dirtiness index trajectories. Note that both trajectories converge to the same value in the long run, which is independent of $\lambda$. However, the dirtiness index is increasing with the altruistic parameter during the first periods: when the central planner maximizes total utility, the economy optimally chooses to devote less resources to abatement activities and therefore a higher dirtiness

\footnotetext{
${ }^{8}$ In an endogenous growth model, Boucekkine, Martinez and Ruiz-Tamarit (2013) find that the short and long run trajectories of the economic variables involved in the Lucas-Uzawa model depend on the altruism parameter and hence it has a positive effect on the long run growth rate of the economy.
} 

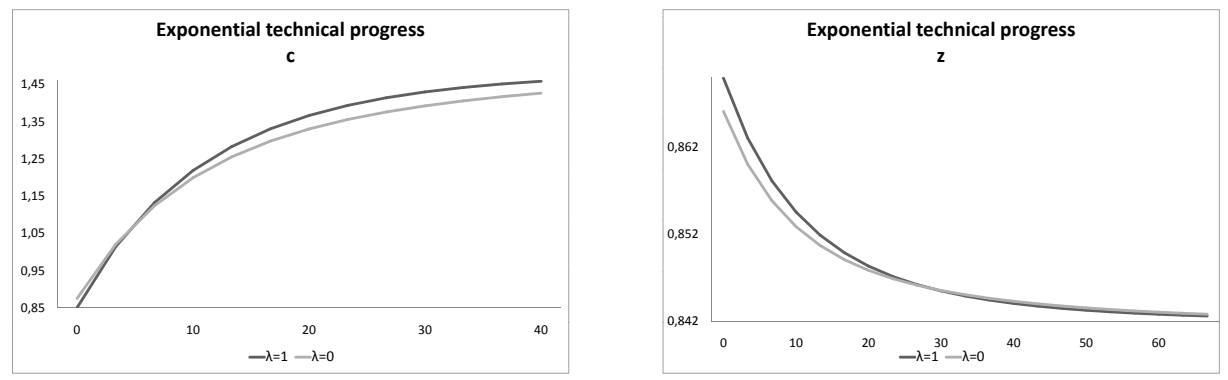

Figure 4. Consumption per capita and the dirtiness index under exponential technical progress.

index in the short run. As a result, per capita consumption is greater in the altruistic economy than in the selfish one, except during the first periods. The previous results are independent of the law of motion of technical progress. It is important to notice that the differences founded are slight even when comparing the two extreme cases. So, although these results are not concluding they reveal that the formulation of the utility function (Millian, intermediate or Benthamite ) will play a role in the short term.

\subsection{Demographic scenarios}

Finally, we show how the environmental variables are affected by demographic dynamics. In this numerical experiment, we simulate our calibrated economy along three demographic scenarios based on UN population projections. Our benchmark economy based on the medium UN scenario is compared to the low and high UN scenarios. To isolate the role of the demographic variables on the evolution of the pollution stock and on the dirtiness index, we use the same preference and technological parameter values for the three economies and we set different demographic parameters following the same procedure as before: we fix $N(0)=1$ as the population level in 2000 along the three demographic scenarios and calculate $\bar{N}$ and $\bar{n}$ using the population projections for 2050 in the high and low scenarios. Since the emissions parameter $G^{M}$ has been fixed to obtain an emission-output ratio equal to 0.12 we need to calculate it for the different demographic scenarios As expected, we obtain that the values of the emissions parameter are increasing with the level of population via aggregate output.

The following table shows the demographic parameters values for the three scenarios and the value of the emissions parameter $G^{M}$.

\begin{tabular}{|l|l|l|l|}
\hline & $\bar{N}$ & $\bar{n}$ & $G^{M}$ \\
\hline Low scenario & 1.22 & 0.07 & 2.35 \\
\hline Medium scenario & 1.47 & 0.04 & 2.834 \\
\hline High scenario & 1.75 & 0.03 & 3.37 \\
\hline
\end{tabular}

In figures 5-6, pale grey lines represent the benchmark economy and dark (grey) lines represent the same economy under the low (high) UN scenario. The stock of pollution solution paths obtained for the three scenarios is shown on the left side of figure 5 . It is decreasing with the population size in the short and in the long run, and converges to a value less than the pollution ceiling, $S=457(S=551)$ in the low (medium) scenario. However, things are quite different for the high scenario. In the latter case, the economy reaches the pollution ceiling at $t^{c}=451$ before the long run stock of pollution value had been reached. Without a pollution ceiling, the high scenario would reach a long run stock of pollution 

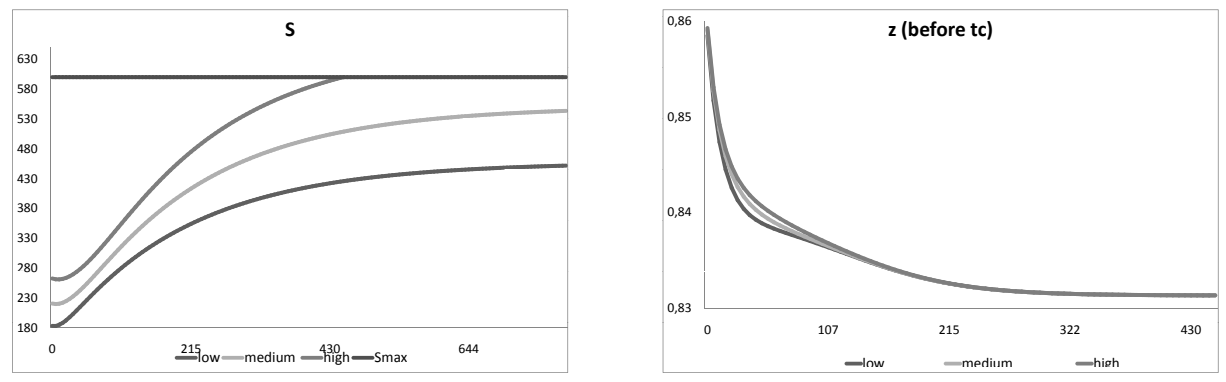

Figure 5. Pollution stock and the dirtiness index for the low, medium and high demographic scenarios.
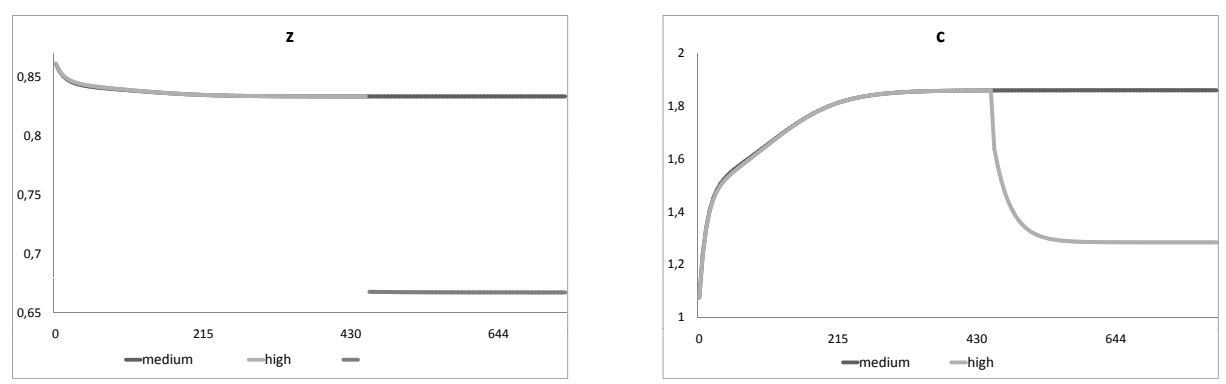

Figure 6. Consumption per capita and the dirtiness index in the medium and high demographic scenarios.

of 656 . Note that $S$ increases with the population size via the regeneration rate and via the emissions parameter. So, as we illustrate in the high scenario, larger populations could generate ecological disasters. As a consequence of the pollution ceiling, beyond $t^{c}$ the stock of pollution keeps constant and equal to $S^{\max }=600$, and the dirtiness index has to be adjusted to keep $S=S^{\max }$.

The evolution of the dirtiness index for the three scenarios before the pollution ceiling is reached in the high scenario is shown on the right side of figure 5. They show a similar pattern with $\mathrm{z}$ increasing with population size during the first periods and then converging to a quite similar value. However, once the pollution ceiling is reached in the high scenario, the evolution of the dirtiness index is completely different: $\mathrm{z}$ has to be adjusted to a lower value in order to keep the stock of pollution to the pollution ceiling level. Figure 6 compares the solution paths of the dirtiness index and per capita consumption in the medium and high scenarios. Again, both variables have a similar dynamics behavior in both scenarios before the pollution ceiling is reached in the high scenario. Then, the decrease in the dirtiness index reflecting that more resources have to be devoted to abatement activities to avoid catastrophe implies less resources devoted to the production side. As a consequence, consumption decreases after $t^{c}$, and converges in the long run to a lower value when we compare it with consumption trajectory in the medium scenario. Again this finding is consistent with the theoretical findings in Section 5.

\section{Conclusion}

In this paper we have studied a prototype of the sustainable growth problem with some salient demographic characteristics. The crucial characteristics of optimal sustainable policies have been singled out, 
first theoretically, then evaluated using a calibrated model. In particular, it has been shown how these policies are affected by actual demographic trends. Of course, this is a first step towards a more comprehensive integration of demographic features into optimal sustainable growth models. An important direction to be taken is to design models in the latter class where population control policies are studied. This requires abandoning the exogenous modelling of demographic dynamics (in this paper, logistic dynamics) and addressing the important issue of optimal population size in connection with the environmental concerns outlined in our benchmark analysis. This is not just adding another control, many pending related ethical issues are on the table. We are currently working along this line.

Acknowledgements. We are grateful to the guest editor and to two anonymous referees whose comments have significanly improved the paper. We also acknowledge the support of the Belgian research programmes ARC on Sustainability as well as the financial support from the Spanish Ministerio de Ciencia e Innovación, Project ECO2010-17943.

\section{References}

[1] J.P. Amigues, M. Moreaux. Optimal Growth under Climate Constraint. Unpublished manuscript, (2013)

[2] J. Aznar-Márquez, J.R. Ruiz-Tamarit. Sufficient and Necessary Conditions for Non-Catastrophic Growth. IRES Discussion Paper, 2012-27, Université Catholique de Louvain, Belgium.

[3] E. Barbier. The Concept of Sustainable Economic Development. Environmental Conservation, 14 (1987), no. 2, 101110 .

[4] R. Boucekkine, N. Hritonenko, Y. Yatsenko. Optimal Investment in Heterogenous Capital and Technology under Restricted Resources. Journal of Optimization Theory and Applications, (2013) forthcoming.

[5] R. Boucekkine, B. Martinez, J.R. Ruiz-Tamarit. Growth vs Level Effect of Population Change on Economic Development: An inspection into human-capital-related mechanisms. Journal of Mathematical Economics, 49 (2013), no. 4, $312-334$.

[6] R. Boucekkine, A. Pommeret, F. Prieur. Optimal Regime Switching and Threshold Effects. Journal of Economic Dynamics and Control, 37 (2013a), no. 12, 2979-2997.

[7] R. Boucekkine, A. Pommeret, F. Prieur. Technological vs Ecological Switch and the Environmental Kuznets Curve. American Journal of Agricultural Economics, 95 (2013b), no. 2, 252-260.

[8] Th. Bréchet, N. Hritonenko, Y. Yatsenko. Adaptation and Mitigation in Long-Term Climate Policy. Environmental and Resource Economics, 55 (2013), no. 2, 217-243.

[9] J.A. Brida, E. Accinelli. The Ramsey Model with Logistic Population Growth. Economic Bulletin, (2007), no. 3(15), $1-8$.

[10] M. M. Byrne. Is Growth a Dirty Word? Pollution, Abatement and Endogenous Growth. Journal of Development Economics, 54 (1997), no. 2, 261-284.

[11] J. Caballé, M. S. Santos. On Endogenous Growth with Physical and Human Capital. Journal of Political Economy, 101 (1993), no. 6, 1042-1067.

[12] Y. S. Chang, S. J. Baek. Limit to Improvement: Myth or Reality? Empirical Analysis of Historical Improvement on three Technologies Influential in the Evolution of Civilization. Technological Forecasting and Social Change, 77 (2010), no. 5, 712-729.

[13] G. Chichilnisky. An Axiomatic Approach to Sustainable Development. Social Choice and Welfare, 13 (1996), no. 2, 231-257.

[14] H. Daly. Towards some Operational Principles of Sustainable Development. Ecological Economics, 2 (1990), no. 1, 1-6.

[15] M. Ferrara, L. Guerrini. The Ramsey Model with Logistic Population Growth and Benthamithe Felicity Function. Proceeding of the 10th WSEAS International Conference on Mathematics and Computers in Business and Economics, Prague, Czech Republic, (2009), March 23-25, 17-20.

[16] B. A. Forster. Optimal Pollution with a non Constant Exponential Rate of Decay. Journal of Environmental Economics and Management, 2 (1975), no. 1, 1-6.

[17] L. Guerrini. The Solow-Swan Model with a Bounded Population Growth Rate. Journal of Mathematical Economics, 42 (2006), no. 1, 14-21.

[18] R. Gradus, S. Smulders. The Trade-off between Environmental Care and Long-term Growth: Pollution in three Prototype Growth Models. Journal of Economics, 58 (1993), no. 1, 25-51.

[19] R. Hartwick. Intergenerational Equity and the Investment of Rents from Exhaustive Resources. American Economic Review, 67 (1977), no. 5, 972-974.

[20] M. Hoel, L. Karp. Taxes versus Quotas for a Stock Pollutant. Resource and Energy Economics, 24 (2002), $367-384$.

[21] Ch. Huang, D. Cai. Constant-Returns Endogenous Growth with Pollution Control. Environmental and Resource Economics, 4 (1994), no. 4, 383-400.

[22] Ph. Michel, G. Rotillon. Disutility of Pollution and Endogenous Growth. Environmental and Resource Economics, 6 (1995), no. 3, 279-300. 
[23] H. Mohtadi. Environment, Growth and Optimal Policy Design. Journal of Public Economics, 63 (1996), no. 1, 119-140.

[24] C. B. Mulligan, X. Sala-i-Martin. Transitional Dynamics in Two-Sector Models of Endogenous Growth. Quarterly Journal of Economics, 108 (1993), no. 3, 739-773.

[25] W. Nordhaus. Rolling the 'DICE': An Optimal Transition Path for Controlling Greenhouse Gases. Environmental and Energy Economics, 15 (1993), no. 1, 27-50.

[26] J. C. V. Pezzey. Sustainability: an Interdisciplinary Guide. Environmental Values, 1 (1992), 321-362.

[27] F. Prieur. The Environmental Kuznets Curve in a World of Irreversibility. Economic Theory, 40 (2009), no. 1, 57-90.

[28] A. B. Reis. Endogenous Growth and the Possibility of Eliminating Pollution. Journal of Environmental Economics and Management, 42 (2001), no. 3, 360-373.

[29] A. Seierstad, K. Sydsaeter. Optimal Control Theory with Economic Applications. Elsevier Science, Amsterdam, 1987.

[30] R. Solow. Sustainability: An economist's perspective. Lecture given at Woods Hole, Massachusetts, 1991. Reprinted in R. Dorfman \& N. S. Dorfman (Eds.), Economics of the Environment, Selected Reading. New York: WWW Norton, 179-187, 1993.

[31] R. Solow. The Economics of Resources or the Resources of Economics. American Economic Review, Papers and Proceedings, 64 (1974), no. 2, 1-14.

[32] N. L. Stokey. Are there Limits to Growth? International Economic Review, 39 (1998), no. 1, 1-31.

[33] O. Tahvonen, C. Withagen. Optimality of Irreversible Pollution Accumulation. Journal of Economic Dynamics and Control, 20 (1996), no. 9.10, 1775-1795.

[34] O. Tahvonen, J. Kuuluvainen. Economic Growth, Pollution and Renewable Resources. Journal of Environmental Economics and Management, 24 (1993), no. 2, 101-118.

[35] Y, Tsur, A. Zemel. Optimal Transition to Backstop Substitutes for Nonrenewable Resources. Journal of Economic Dynamics and Control, 27 (2003), no. 4, 551-572.

[36] UNPD. World Population to 2300. New York: United Nations Population Division, 2004.

[37] M. L. Weitzman. A Review of the Stern Review on the Economics of Climate Change. Journal of Economic Literature, 45 (2007), no. 3, 703-724.

[38] C. Withagen. Pollution and Exhaustibility of Fossil Fuels. Resources and Energy Economics, 16 (1994), no. 3, $235-242$. 